\title{
Article \\ The Uniaxial Limit of the Non-Inertial Qian-Sheng Model for Liquid Crystals
}

\author{
Sirui $\mathrm{Li}^{1,2, *(1)}$ and Fangxin Zhao ${ }^{2}$ \\ 1 School of Mathematical Sciences, Zhejiang University, Hangzhou 310027, China \\ 2 School of Mathematics and Statistics, Guizhou University, Guiyang 550025, China; fxzhaomath@163.com \\ * Correspondence: srli@gzu.edu.cn
}

Citation: Li, S.; Zhao, F. The Uniaxial Limit of the Non-Inertial Qian-Sheng Model for Liquid Crystals. Mathematics 2021, 9, 912. https:// doi.org/10.3390/math9080912

Academic Editor: Athanasios Tzavaras

Received: 10 March 2021

Accepted: 13 April 2021

Published: 20 April 2021

Publisher's Note: MDPI stays neutral with regard to jurisdictional claims in published maps and institutional affiliations.

Copyright: (c) 2021 by the authors. Licensee MDPI, Basel, Switzerland. This article is an open access article distributed under the terms and conditions of the Creative Commons Attribution (CC BY) license (https:// creativecommons.org/licenses/by/ $4.0 /)$.

\begin{abstract}
In this article, we consider the Qian-Sheng model in the Landau-de Gennes framework describing nematic liquid crystal flows when the inertial effect is neglected. By taking the limit of elastic constant to zero (also called the uniaxial limit) and utilizing the so-called Hilbert expansion method, we provide a rigorous derivation from the non-inertial Qian-Sheng model to the EricksenLeslie model.
\end{abstract}

Keywords: liquid crystals; Qian-Sheng model; Ericksen-Leslie model; uniaxial limit

\section{Introduction}

Liquid crystals feature partial order (also called the local anisotropy), which results in physical properties between liquid and solid. Nematic order is the simplest kind of orientational order, with the molecules displaying a long-range orientation but randomly distributing in space. Roughly speaking, there exists three different but closely related models to describe the dynamical behavior of nematic liquid crystals: the Doi-Onsager model, the Ericksen-Leslie model and the Landau-de Gennes model. The first one is based on microscopic statistical mechanics. The latter two are based on macroscopic continuum mechanics.

Exploring relationships between different dynamical models is a fundamental subject in the theoretical study of liquid crystals. In this respect, the formal derivations were constructed by Kuzzu-Doi [1] and E-Zhang [2] from the Doi-Onsager model to the Ericksen-Leslie model. By the Hilbert expansion, the rigorous convergence result for smooth evolution from the Doi-Onsager model to the Ericksen-Leslie model was subsequently established by Wang-Zhang-Zhang [3] under the small Deborah number limit. By means of carefully analyzing the properties of the linearized operators, rigorous verification given in [3] circumvent essential difficulties from the uniform control for the singular terms with respect to a small parameter. Following the spirit of [3], Li-Wang-Zhang [4] provided a strict derivation from the molecular-based $Q$-tensor dynamical model, obtained from the molecular kinetic theory by the Bingham closure, to the Ericksen-Leslie model. Similar rigorous convergence results were obtained by Wang et al. [5] for the Beris-Edwards model in the framework of smooth solutions. A unified formulation for liquid crystal modeling was proposed by Han et al. [6] to establish relations between microscopic theory and macroscopic theory.

The main aim of this article is to rigorously justify the uniaxial limit from the noninertial Qian-Sheng model in Landau-de Gennes framework into the Ericksen-Leslie model away from singularities of the solution. Concerning the inertial Qian-Sheng model, which is the hyperbolic-parabolic system, the corresponding uniaxial limit has been rigorously proved by the first author and coauthor in [7]. The Qian-Sheng model is a representative dynamical model in the Landau-de Gennes framework, and the small inertial term is usually neglected on physical grounds(see [8,9] and so on). For the case of the inertial coefficient being zero, this article provides a valuable supplement to the singular limit 
problem of the $Q$-tensor dynamical model for liquid crystals. Similar to the idea in $[5,7]$, to control the singular terms in the equation for remainders, we also need to deeply explore the cancellation relation and symmetric structures of the equations. What follows is a brief overview of two macroscopic continuum models concerned in this article.

The Ericksen-Leslie model, established by Ericksen [10] and Leslie [11] in the 1960s, is a system coupling the Navier-Stokes equation of the fluid velocity $\mathbf{v}=\mathbf{v}(\mathbf{x}, t)$ with the evolution equation of the director field $\mathbf{n}=\mathbf{n}(\mathbf{x}, t) \in \mathbb{S}^{2}$. Taking no account of inertial effects, the Ericksen-Leslie model can be given as follows:

$$
\begin{aligned}
& \frac{\partial \mathbf{v}}{\partial t}+\mathbf{v} \cdot \nabla \mathbf{v}=-\nabla p+\nabla \cdot \sigma, \\
& \nabla \cdot \mathbf{v}=0, \\
& \mathbf{n} \times\left(\mathbf{h}-\gamma_{1} \mathbf{N}-\gamma_{2} \mathbf{D} \cdot \mathbf{n}\right)=0,
\end{aligned}
$$

where $\mathbf{v}$ is the fluid velocity and $p$ the pressure, the stress $\sigma=\sigma^{L}+\sigma^{E}$ and the molecular field $\mathbf{h}$ are respectively defined by:

$$
\begin{aligned}
\sigma^{L} & =\alpha_{1}(\mathbf{n n}: \mathbf{D}) \mathbf{n n}+\alpha_{2} \mathbf{n} \mathbf{N}+\alpha_{3} \mathbf{N n}+\alpha_{4} \mathbf{D}+\alpha_{5} \mathbf{n n} \cdot \mathbf{D}+\alpha_{6} \mathbf{D} \cdot \mathbf{n n}, \\
\sigma^{E} & =-\frac{\partial E_{F}}{\partial(\nabla \mathbf{n})} \cdot(\nabla \mathbf{n})^{T}, \\
\mathbf{h} & =-\frac{\delta E_{F}}{\delta \mathbf{n}}=-\frac{\partial E_{F}}{\partial \mathbf{n}}+\nabla \cdot \frac{\partial E_{F}}{\partial(\nabla \mathbf{n})} .
\end{aligned}
$$

Here the Oseen-Frank energy $E_{F}$ is given by

$$
\begin{aligned}
E_{F}(\mathbf{n}, \nabla \mathbf{n})= & \frac{k_{1}}{2}(\nabla \cdot \mathbf{n})^{2}+\frac{k_{2}}{2}(\mathbf{n} \cdot(\nabla \times \mathbf{n}))^{2}+\frac{k_{3}}{2}|\mathbf{n} \times(\nabla \times \mathbf{n})|^{2} \\
& +\frac{k_{2}+k_{4}}{2}\left(\operatorname{tr}(\nabla \mathbf{n})^{2}-(\nabla \cdot \mathbf{n})^{2}\right),
\end{aligned}
$$

where $k_{1}, k_{2}, k_{3}, k_{4}$ are the Frank constants. In addition, $\mathbf{D}=\frac{1}{2}\left((\nabla \mathbf{v})^{T}+\nabla \mathbf{v}\right)$ and $\mathbf{\Omega}=\frac{1}{2}\left(\nabla \mathbf{v}-(\nabla \mathbf{v})^{T}\right)$ stand for the rate of strain tensor and the vorticity tensor, respectively. While $\mathbf{N}=\mathbf{n}_{t}+\mathbf{v} \cdot \nabla \mathbf{n}-\mathbf{\Omega} \cdot \mathbf{n}$ is called the co-rotational time flux of the director $\mathbf{n}$.

The six constants $\alpha_{1}, \cdots, \alpha_{6}$ in (4) are called the Leslie viscosity coefficients. They and the coefficients $\gamma_{1}, \gamma_{2}$ together fulfil the following relations:

$$
\begin{aligned}
& \alpha_{2}+\alpha_{3}=\alpha_{6}-\alpha_{5}, \\
& \gamma_{1}=\alpha_{3}-\alpha_{2}, \quad \gamma_{2}=\alpha_{6}-\alpha_{5} .
\end{aligned}
$$

The relations (7) and (8) guarantee the dissipative character of the system (1)-(3), i.e.,

$$
\begin{aligned}
-\frac{\mathrm{d}}{\mathrm{d} t}\left(\int_{\mathbb{R}^{3}} \frac{1}{2}|\mathbf{v}|^{2} \mathrm{~d} \mathbf{x}+E_{F}\right)=\int_{\mathbb{R}^{3}} & \left(\left(\alpha_{1}+\frac{\gamma_{2}^{2}}{\gamma_{1}}\right)(\mathbf{D}: \mathbf{n n})^{2}+\alpha_{4}|\mathbf{D}|^{2}\right. \\
& \left.+\left(\alpha_{5}+\alpha_{6}-\frac{\gamma_{2}^{2}}{\gamma_{1}}\right)|\mathbf{D} \cdot \mathbf{n}|^{2}+\frac{1}{\gamma_{1}}|\mathbf{n} \times \mathbf{h}|^{2}\right) \mathrm{d} \mathbf{x} .
\end{aligned}
$$

For the Ericksen-Leslie model, there has been published much analytic work. We only recall some relevant results here. For the non-inertial version, the well-posedness results can be referred to [12-15] and references therein. Concerning the inertial (hyperbolic) version, the well-posedness of classical solutions was studied in [16-18].

We next introduce the hydrodynamical model of liquid crystals based on the Landaude Gennes theory. This theory employs a symmetric traceless $Q$-tensor to characterize the 
alignments of molecules. The tensor $Q(\mathbf{x})$ can be interpreted as the second-order moment of the density distribution function $f$,

$$
Q(\mathbf{x})=\int_{\mathbb{S}^{2}}\left(\mathbf{m} \mathbf{m}-\frac{1}{3} \mathbf{I}\right) f(\mathbf{x}, \mathbf{m}) \mathrm{d} \mathbf{m} .
$$

For a symmetric traceless tensor $Q(\mathbf{x})$, one can find $s(\mathbf{x}), r(\mathbf{x}) \in \mathbb{R}, \mathbf{n}, \mathbf{m} \in \mathbb{S}^{2}$ with $\mathbf{n} \cdot \mathbf{m}=0$ such that

$$
Q(\mathbf{x})=s(\mathbf{x})\left(\mathbf{n n}-\frac{1}{3} \mathbf{I}\right)+r(\mathbf{x})\left(\mathbf{m m}-\frac{1}{3} \mathbf{I}\right)
$$

where $\mathbf{I}$ is a $3 \times 3$ identity matrix. Liquid crystals are said to be isotropic when $s=r=0$, uniaxial when $s \neq 0, r=0$, and biaxial when $s \neq 0, r \neq 0$.

The general Landau-de Gennes energy functional is given as follows:

$$
\begin{aligned}
& \mathcal{F}(Q, \nabla Q) \\
& =\int_{\mathbb{R}^{3}}\left\{-\frac{a}{2} \operatorname{Tr}\left(Q^{2}\right)-\frac{b}{3} \operatorname{Tr}\left(Q^{3}\right)+\frac{c}{4}\left(\operatorname{Tr}\left(Q^{2}\right)^{2}\right.\right. \\
& \left.\quad+\frac{1}{2}\left(L_{1}|\nabla Q|^{2}+L_{2} Q_{i j, j} Q_{i k, k}+L_{3} Q_{i j, k} Q_{i k, j}+L_{4} Q_{i j} Q_{k l, i} Q_{k l, j}\right)\right\} \mathrm{d} \mathbf{x} \\
& \stackrel{\text { def }}{=} \int_{\mathbb{R}^{3}}\left(F_{b}(Q)+F_{e}(Q, \nabla Q)\right) \mathrm{d} \mathbf{x},
\end{aligned}
$$

where $\mathbf{x}$ is the material point in $\mathbb{R}^{3}$, the coefficients $a, b, c$ are non-negative bulk constants depending on the temperature and material, and $L_{i}(i=1,2,3,4)$ are material-dependent elastic constants. In (12), $F_{b}(Q)$ is the bulk energy characterizing the isotropic-nematic phase transition, while $F_{e}(Q, \nabla Q)$ is the elastic energy describing the distortion effect. More details can be referred to $[19,20]$.

In the Landau-de Gennes framework, there are two representative $Q$-tensor dynamical models: the Beris-Edwards model [21] and the Qian-Sheng model [22], which can be directly derived by the variational method. The well-posedness results of the BerisEdwards model on whole space and bounded domain can be referred to [23-28], respectively. The well-posedness results for the inertial Qian-Sheng model and the non-inertial version can be found in [29-31], respectively.

In this article, we will be concerned with the following $Q$-tensor hydrodynamical system, proposed by T. Qian and P. Sheng in [22], with taking no account of the inertial effect:

$$
\begin{aligned}
\frac{\partial Q}{\partial t}+\mathbf{v} \cdot \nabla Q & =\frac{1}{\mu_{1}} \mathbf{H}+\chi \mathbf{D}+\mathbf{\Omega} \cdot Q-Q \cdot \mathbf{\Omega}, \\
\frac{\partial \mathbf{v}}{\partial t}+\mathbf{v} \cdot \nabla \mathbf{v} & =-\nabla p+\nabla \cdot\left(\sigma^{\mathcal{s}}+\sigma^{a}+\sigma^{d}\right), \\
\nabla \cdot \mathbf{v} & =0,
\end{aligned}
$$

where $\Omega \cdot Q-Q \cdot \Omega$ are induced by the rotation part of the velocity gradient, the molecular field $\mathbf{H}$ is given by

$$
\mathbf{H}=-\frac{\delta \mathcal{F}(Q, \nabla Q)}{\delta Q} .
$$

$\sigma_{i j}^{d}=-\frac{\partial \mathcal{F}}{\partial Q_{k l, j}} \partial_{i} Q_{k l}$ is the distortion stress, $\sigma^{a}=Q \cdot \mathbf{H}-\mathbf{H} \cdot Q$ is the anti-symmetric stress, and $\sigma^{s}$ is the symmetric stress defined as

$$
\sigma^{\mathcal{S}}=\chi \mathbf{H}+\beta_{1}^{\prime} Q(Q: \mathbf{D})+\beta_{2}^{\prime} \mathbf{D}+\beta_{3}^{\prime}(Q \cdot \mathbf{D}+\mathbf{D} \cdot Q) .
$$


While the viscosity coefficients are expressed as

$$
\chi=-\frac{1}{2} \frac{\mu_{2}}{\mu_{1}}, \beta_{1}^{\prime}=\beta_{1}, \beta_{2}^{\prime}=\beta_{4}-\frac{\mu_{2}^{2}}{4 \mu_{1}}, \beta_{3}^{\prime}=\frac{\beta_{5}+\beta_{6}}{2} .
$$

Here $\beta_{1}, \beta_{4}, \beta_{5}, \beta_{6}, \mu_{1}$ and $\mu_{2}$ are viscosity coefficients in the original Qian-Sheng model and satisfy the following Parodi's relation [32]:

$$
\beta_{6}-\beta_{5}=\mu_{2}
$$

In order to maintain the energy dissipation law of the system (13)-(15), we assume that the viscosity coefficients $\chi, \mu_{1}, \beta_{1}^{\prime}, \beta_{2}^{\prime}$ are positive constants, $\mu_{2}<0$ and $\beta_{2}^{\prime}>\max \left\{\beta_{1}^{\prime}, \beta_{3}^{\prime}\right\}$. This assumptions have the physical meanings. For instance, the relations between the coefficients corresponding to the classical physical material, MBBA (4-methoxybenzyliden4'-butylanilin), are given in [9] by

$$
\frac{\mu_{2}}{\mu_{1}} \approx-1.92, \frac{\beta_{1}}{\mu_{1}} \approx 0.17, \frac{\beta_{4}}{\mu_{1}} \approx 1.99, \frac{\beta_{5}}{\mu_{1}} \approx 0.70, \frac{\beta_{6}}{\mu_{1}} \approx-0.79 .
$$

More specific assumptions on the viscosity coefficients can be also found in [8].

The article is organized as follows. In Section 2, we present some important properties of the linearized operators which will be used in deriving the Ericksen-Leslie model from the non-inertial Qian-Sheng model. Meanwhile, the main result of this article is stated. In Section 3, by making the so-called Hilbert expansion, we present a rigorous derivation from the non-inertial Qian-Sheng model to the Ericksen-Leslie model.

Notations and Conventions: The Einstein summation convention is employed in this article. The space of symmetric traceless tensors is defined as:

$$
\mathbb{M}_{0}^{3} \stackrel{\text { def }}{=}\left\{Q \in \mathbb{R}^{3 \times 3}: Q^{T}=Q, \operatorname{Tr}(Q)=0\right\},
$$

which is endowed with the inner product $\left\langle Q_{1}, Q_{2}\right\rangle \stackrel{\text { def }}{=} \int_{\mathbb{R}^{3}} Q_{1}(\mathbf{x}): Q_{2}(\mathbf{x}) \mathrm{d} \mathbf{x}$. The set $\mathbb{M}_{0}^{3}$ is a five-dimensional linear subspace of $\mathbb{R}^{3 \times 3}$. The matrix norm on $\mathbb{M}_{0}^{3}$ is defined as $|Q| \stackrel{\text { def }}{=} \sqrt{\operatorname{tr} Q^{2}}=\sqrt{Q_{i j} Q_{i j}}$. For two tensors $A, B \in \mathbb{M}_{0}^{3}$, we denote $(A \cdot B)_{i j}=A_{i k} B_{k j}$ and $A: B=A_{i j} B_{i j}$. In addition, $\mathbf{n}_{1} \otimes \mathbf{n}_{2}$ denotes the tensor product of two vectors $\mathbf{n}_{1}, \mathbf{n}_{2}$, and we usually omit the symbol $\otimes$ for simplicity. We use $f_{i}$ to denote $\partial_{i} f$ and $\mathbf{I}$ to denote the $3 \times 3$ order identity tensor.

\section{Preliminaries and Main Results}

In this section, we first recall some results from [5] concerning critical points and the linearized operator, which will play a key role in deriving the Ericksen-Lesile model from the non-inertial Qian-Sheng model. The main result of this article is subsequently stated.

In physics, in contrast to the bulk constants $a, b, c$, the elastic coefficients $L_{i}(i=1,2,3,4)$ are usually regarded as very small parameters, we thus give the Landau-de Gennes energy functional (12) with a small parameter $\varepsilon$ :

$$
\mathcal{F}_{\varepsilon}(Q, \nabla Q)=\int_{\mathbb{R}^{3}}\left(\frac{1}{\varepsilon} F_{b}(Q)+F_{e}(Q, \nabla Q)\right) \mathrm{d} \mathbf{x},
$$

where $a, b, c, L_{i}(i=1,2,3) \sim O(1)$. When the small elastic parameter $\varepsilon \rightarrow 0$, then $Q^{\varepsilon} \rightarrow$ $Q_{0}=s\left(\mathbf{n n}-\frac{1}{3} \mathbf{I}\right)$, i.e., the corresponding liquid crystal system will converge to a unixial state in which molecules tend to align in a preferred direction. We assume that the elastic coefficients $L_{i}(i=1,2,3,4)$ satisfy

$$
L_{1}>0, L_{1}+L_{2}+L_{3}>0, L_{4}=0 .
$$


Under the assumption (20), we see from Lemma 2.5 in [5] that the elastic energy is strictly positive, i.e., there exists some constant $L_{0}=L_{0}\left(L_{1}, L_{2}, L_{3}\right)>0$ such that

$$
\int_{\mathbb{R}^{3}} \mathscr{L}(Q): Q \mathrm{~d} \mathbf{x} \geq L_{0}\|\nabla Q\|_{L^{2}}^{2}
$$

where the definition of the operator $\mathscr{L}$ will be given below.

In this article, we primarily investigate the singular limit of the non-inertial QianSheng model with a small parameter $\varepsilon$ :

$$
\begin{aligned}
\frac{\partial Q^{\varepsilon}}{\partial t}+\mathbf{v}^{\varepsilon} \cdot \nabla Q^{\varepsilon} & =\frac{1}{\mu_{1}} \mathbf{H}^{\varepsilon}+\chi \mathbf{D}^{\varepsilon}+\mathbf{\Omega}^{\varepsilon} \cdot Q^{\varepsilon}-Q^{\varepsilon} \cdot \mathbf{\Omega}^{\varepsilon}, \\
\frac{\partial \mathbf{v}^{\varepsilon}}{\partial t}+\mathbf{v}^{\varepsilon} \cdot \nabla \mathbf{v}^{\varepsilon} & =-\nabla p^{\varepsilon}+\nabla \cdot\left(\sigma_{\varepsilon}^{\mathcal{s}}+\sigma_{\varepsilon}^{a}+\sigma_{\varepsilon}^{d}\right), \\
\nabla \cdot \mathbf{v}^{\varepsilon} & =0
\end{aligned}
$$

where $\mathbf{D}^{\varepsilon}=\frac{1}{2}\left(\nabla \mathbf{v}^{\varepsilon}+\left(\nabla \mathbf{v}^{\varepsilon}\right)^{T}\right), \mathbf{\Omega}^{\varepsilon}=\frac{1}{2}\left(\nabla \mathbf{v}^{\varepsilon}-\left(\nabla \mathbf{v}^{\varepsilon}\right)^{T}\right)$, and

$$
\begin{aligned}
& \sigma_{\varepsilon}^{\varsigma}=\chi \mathbf{H}^{\varepsilon}+\beta_{1}^{\prime} Q^{\varepsilon}\left(Q^{\varepsilon}: \mathbf{D}^{\varepsilon}\right)+\beta_{2}^{\prime} \mathbf{D}^{\varepsilon}+\beta_{3}^{\prime}\left(Q^{\varepsilon} \cdot \mathbf{D}^{\varepsilon}+\mathbf{D}^{\varepsilon} \cdot Q^{\varepsilon}\right), \\
& \sigma_{\varepsilon}^{a}=Q^{\varepsilon} \cdot \mathbf{H}^{\varepsilon}-\mathbf{H}^{\varepsilon} \cdot Q^{\varepsilon}, \quad\left(\sigma_{\varepsilon}^{d}\right)_{j i}=-\frac{\partial \mathcal{F}_{\varepsilon}}{\partial Q_{k l, j}^{\varepsilon}} Q_{k l, i}^{\varepsilon} \stackrel{\text { def }}{=} \sigma^{d}\left(Q^{\varepsilon}, Q^{\varepsilon}\right) .
\end{aligned}
$$

The molecular field $\mathbf{H}^{\varepsilon}$ is defined by

$$
\mathbf{H}^{\varepsilon}(Q)=-\frac{1}{\varepsilon} \frac{\partial F_{b}}{\partial Q}+\partial_{i}\left(\frac{\partial F_{e}}{\partial Q_{, i}}\right) \stackrel{\text { def }}{=}-\frac{1}{\varepsilon} \mathscr{T}(Q)-\mathscr{L}(Q),
$$

where two operators $\mathscr{T}$ and $\mathscr{L}$ are respectively expressed as

$$
\begin{aligned}
\mathscr{T}(Q) & =-a Q-b Q^{2}+c|Q|^{2} Q+\frac{1}{3} b|Q|^{2} \mathbf{I}, \\
(\mathscr{L}(Q))_{k l} & =-\left(L_{1} \Delta Q_{k l}+\frac{1}{2}\left(L_{2}+L_{3}\right)\left(Q_{k m, m l}+Q_{l m, m k}-\frac{2}{3} \delta_{k l} Q_{i j, i j}\right)\right) .
\end{aligned}
$$

\subsection{Critical Points and the Linearized Operator}

We first provide the definition of critical points for the bulk energy $F_{b}(Q)$. A tensor $Q_{0}$ is called a critical point of $F_{b}(Q)$ if $\mathscr{T}\left(Q_{0}\right)=0$. The following characterization of critical points can be seen from $[5,33]$.

Proposition 1. $\mathscr{T}(Q)=0$ if and only if $Q=s\left(\mathbf{n n}-\frac{1}{3} \mathbf{I}\right)$ for some $\mathbf{n} \in \mathbb{S}^{2}$, where $s=0$ or $s$ is a solution of $2 c s^{2}-b s+3 a=0$, that is,

$$
s_{1}=\frac{b+\sqrt{b^{2}+24 a c}}{4 c}, s_{2}=\frac{b-\sqrt{b^{2}+24 a c}}{4 c} .
$$

Moreover, the critical point $Q_{0}=s\left(\mathbf{n n}-\frac{1}{3} \mathbf{I}\right)$ is stable if $s=s_{1}$.

Given a critical point $Q_{0}$, the linearized operator $\mathscr{H}_{Q_{0}}$ of $\mathscr{T}(Q)$ around $Q_{0}$ is defined by

$$
\mathscr{H}_{Q_{0}}(Q)=-a Q-b\left(Q_{0} \cdot Q+Q \cdot Q_{0}\right)+c\left|Q_{0}\right|^{2} Q+2\left(Q_{0}: Q\right)\left(c Q_{0}+\frac{b}{3} \mathbf{I}\right) .
$$


Then a direct calculation yields

$$
\begin{aligned}
\mathscr{H}_{Q_{0}}(Q)= & b s\left(Q-(\mathbf{n n} \cdot Q+Q \cdot \mathbf{n n})+\frac{2}{3}(Q: \mathbf{n n}) \mathbf{I}\right) \\
& +2 c s^{2}(Q: \mathbf{n n})\left(\mathbf{n n}-\frac{1}{3} \mathbf{I}\right) \\
\stackrel{\text { def }}{=} & \mathscr{H}_{\mathbf{n}}(Q) .
\end{aligned}
$$

The kernel space of the linearized operator $\mathscr{H}_{\mathbf{n}}$, being a two-dimensional subspace of $\mathbb{M}_{0}^{3}$, can be defined as

$$
\operatorname{Ker} \mathscr{H}_{\mathbf{n}} \stackrel{\text { def }}{=}\left\{\mathbf{n n}^{\perp}+\mathbf{n}^{\perp} \mathbf{n} \in \mathbb{M}_{0}^{3}: \mathbf{n}^{\perp} \in \mathbb{V}_{\mathbf{n}}\right\}
$$

for any given $\mathbf{n} \in \mathbb{S}^{2}$, where $\mathbb{V}_{\mathbf{n}} \stackrel{\text { def }}{=}\left\{\mathbf{n}^{\perp} \in \mathbb{R}^{3}: \mathbf{n}^{\perp} \cdot \mathbf{n}=0\right\}$ and $\mathbf{n}^{\perp}$ represents a unit vector perpendicular to $\mathbf{n}$. Let $\mathscr{P}^{\text {in }}$ be the projection operator from $\mathbb{M}_{0}^{3}$ into Ker $\mathscr{H}_{\mathbf{n}}$ and $\mathscr{P}$ out the projection operator from $\mathbb{M}_{0}^{3}$ into $\left(\operatorname{Ker} \mathscr{H}_{\mathbf{n}}\right)^{\perp}$. The projection operators $\mathscr{P}^{\text {in }}$ and $\mathscr{P}^{\text {out }}$ (see the definitions in [5]) can be respectively expressed as

$$
\begin{aligned}
\mathscr{P}^{\text {in }}(Q) & =(\mathbf{n n} \cdot Q+Q \cdot \mathbf{n n})-2(Q: \mathbf{n n}) \mathbf{n n}, \\
\mathscr{P}^{\text {out }}(Q) & =Q-(\mathbf{n n} \cdot Q+Q \cdot \mathbf{n n})+2(Q: \mathbf{n n}) \mathbf{n n} .
\end{aligned}
$$

The important properties of the linearized operator $\mathscr{H}_{\mathbf{n}}$ obtained in [5] are as follows:

Proposition 2. (i) For any $\mathbf{n} \in \mathbb{S}^{2}$, it holds that $\mathscr{H}_{\mathbf{n}} \operatorname{Ker} \mathscr{H}_{\mathbf{n}}=0$, i.e., $\mathscr{H}_{\mathbf{n}}(Q) \in\left(\operatorname{Ker} \mathscr{H}_{\mathbf{n}}\right)^{\perp}$. (ii) There exists a positive constant $C_{0}=c_{0}(a, b, c)>0$ such that for any $Q \in\left(\operatorname{Ker} \mathscr{H}_{\mathbf{n}}\right)^{\perp}$,

$$
\mathscr{H}_{\mathbf{n}}(Q): Q \geq c_{0}|Q|^{2} .
$$

(iii) $\mathscr{H}_{\mathbf{n}}$ is a 1-1 map on $\left(\operatorname{Ker} \mathscr{H}_{\mathbf{n}}\right)^{\perp}$ and its inverse $\mathscr{H}_{\mathbf{n}}^{-1}$ is given by

$$
\begin{aligned}
\mathscr{H}_{\mathbf{n}}^{-1}(Q)= & \frac{1}{b s}\left(Q-(\mathbf{n n} \cdot Q+Q \cdot \mathbf{n n})+\frac{2}{3}(Q: \mathbf{n n}) \mathbf{I}\right) \\
& +\frac{4 b+2 c s}{b s(4 c s-b)}(Q: \mathbf{n n})\left(\mathbf{n n}-\frac{1}{3} \mathbf{I}\right) .
\end{aligned}
$$

\subsection{The Main Result}

We define the viscosity coefficients in the Ericksen-Leslie model as:

$$
\begin{aligned}
& \alpha_{1}=\beta_{1}^{\prime} s^{2}, \quad \alpha_{2}=-\left(s \chi+s^{2}\right) \mu_{1}, \quad \alpha_{3}=\left(-s \chi+s^{2}\right) \mu_{1}, \\
& \alpha_{4}=\mu_{1} \chi^{2}+\beta_{2}^{\prime}-\frac{2}{3} s \beta_{3}^{\prime}, \quad \alpha_{5}=s \beta_{3}^{\prime}+\mu_{1} s \chi, \quad \alpha_{6}=s \beta_{3}^{\prime}-\mu_{1} s \chi,
\end{aligned}
$$

and the coefficients $\gamma_{1}$ and $\gamma_{2}$ are

$$
\gamma_{1}=2 \mu_{1} s^{2}, \quad \gamma_{2}=-2 \mu_{1} s \chi .
$$

In addition, the elastic constants in the Oseen-Frank energy are given by

$$
k_{1}=k_{3}=2\left(L_{1}+L_{2}+L_{3}\right) s^{2}, \quad k_{2}=2 L_{1} s^{2}, \quad k_{4}=L_{3} s^{2} .
$$

The main result of this article is stated as follows. 
Theorem 1. Assume $Q_{0}(t, \mathbf{x})=s\left(\mathbf{n}(t, \mathbf{x}) \mathbf{n}(t, \mathbf{x})-\frac{1}{3} \mathbf{I}\right)$ is a stable critical point of the bulk energy $F_{b}(Q)$. Let $(\mathbf{n}(t, \mathbf{x}), \mathbf{v}(t, \mathbf{x}))$ be a smooth solution of the Ericksen-Leslie model (1)-(3) on $[0, T]$ with the coefficients given by (27)-(29), which satisfies

$$
\mathbf{v} \in C\left([0, T] ; H^{k}\right), \quad \nabla \mathbf{n} \in C\left([0, T] ; H^{k}\right) \quad \text { for } \quad k \geq 20 .
$$

Suppose that the initial data $\left(Q_{I}^{\varepsilon}, \mathbf{v}_{I}^{\varepsilon}\right)$ takes the form

$$
Q_{I}^{\varepsilon}(\mathbf{x})=\sum_{k=0}^{3} \varepsilon^{k} Q_{k}(0, \mathbf{x})+\varepsilon^{3} Q_{I, R}^{\varepsilon}(\mathbf{x}), \quad \mathbf{v}_{I}^{\varepsilon}(\mathbf{x})=\sum_{k=0}^{2} \varepsilon^{k} \mathbf{v}_{k}(0, \mathbf{x})+\varepsilon^{3} \mathbf{v}_{I, R}^{\varepsilon}(\mathbf{x}),
$$

where the functions $\left(Q_{1}, Q_{2}, Q_{3}, \mathbf{v}_{1}, \mathbf{v}_{2}\right)$ are determined by Proposition 4 , and $\left(Q_{I, R}^{\varepsilon}, \mathbf{v}_{I, R}^{\varepsilon}\right)$ fulfils

$$
\left\|\mathbf{v}_{I, R}^{\varepsilon}\right\|_{H^{2}}+\left\|Q_{I, R}^{\varepsilon}\right\|_{H^{3}}+\varepsilon^{-1}\left\|\mathscr{P}^{\text {out }}\left(Q_{I, R}^{\varepsilon}\right)\right\|_{L^{2}} \leq E_{0} .
$$

Then there exists $\varepsilon_{0}>0$ and $E_{1}>0$ such that for all $\varepsilon<\varepsilon_{0}$, the non-inertial Qian-Sheng model (21)-(23) has a unique solution $\left(Q^{\varepsilon}(t, \mathbf{x}), \mathbf{v}^{\varepsilon}(t, \mathbf{x})\right)$ on $[0, T]$ that has the Hilbert expansion

$$
Q^{\varepsilon}(t, \mathbf{x})=\sum_{k=0}^{3} \varepsilon^{k} Q_{k}(t, \mathbf{x})+\varepsilon^{3} Q_{R}^{\varepsilon}(t, \mathbf{x}), \mathbf{v}^{\varepsilon}(t, \mathbf{x})=\sum_{k=0}^{2} \varepsilon^{k} \mathbf{v}_{k}(t, \mathbf{x})+\varepsilon^{3} \mathbf{v}_{R}^{\varepsilon}(t, \mathbf{x}),
$$

where, for any $t \in[0, T],\left(Q_{R}^{\varepsilon}, \mathbf{v}_{R}^{\varepsilon}\right)$ satisfies

$$
\begin{aligned}
& \left\|\left(Q_{R}^{\varepsilon}, \varepsilon^{-\frac{1}{2}}\left(\mathscr{H}_{\mathbf{n}}^{\varepsilon}\left(Q_{R}^{\varepsilon}\right): Q_{R}^{\varepsilon}\right)^{\frac{1}{2}}, \varepsilon^{\frac{1}{2}}\left(\mathscr{H}_{\mathbf{n}}^{\varepsilon}\left(\partial_{i} Q_{R}^{\varepsilon}\right): \partial_{i} Q_{R}^{\varepsilon}\right)^{1 / 2}, \varepsilon^{\frac{3}{2}}\left(\mathscr{H}_{\mathbf{n}}^{\varepsilon}\left(\Delta Q_{R}^{\varepsilon}\right): \Delta Q_{R}^{\varepsilon}\right)^{\frac{1}{2}}\right)(t)\right\|_{L^{2}} \\
& +\left\|\left(\mathbf{v}_{R}^{\varepsilon}, \varepsilon \nabla \mathbf{v}_{R}^{\varepsilon}, \varepsilon^{2} \Delta \mathbf{v}_{R}^{\varepsilon}\right)(t)\right\|_{L^{2}} \leq E_{1},
\end{aligned}
$$

here $\mathscr{H}_{\mathbf{n}}^{\varepsilon}(Q)=\mathscr{H}_{\mathbf{n}}(Q)+\varepsilon \mathscr{L}(Q)$ and the constant $E_{1}$ is independent of $\varepsilon$.

Remark 1. It is worth pointing out that the relations between the Leslie coefficients (27) are completely in agreement with those given by Qian and Sheng's original work in [22] if taking $s=\frac{3}{2} S_{0}$.

Remark 2. The existence of the Hilbert expansion and the error estimates of remainder equations are closely related to whether the energy of the Ericksen-Leslie model is dissipated or not. Theorem 1 requires that the coefficients $\chi, \mu_{1}, \beta_{1}^{\prime}, \beta_{2}^{\prime}$ are positive constants, $\mu_{2}<0$ and $\beta_{2}^{\prime}>\max \left\{\beta_{1}^{\prime}, \beta_{3}^{\prime}\right\}$, however, we do not assume $\beta_{2}^{\prime}$ is large enough. Proposition 2.2 in [15] tells us that the energy dissipation law (9) holds, that is,

$$
\hat{\beta}_{1}|\mathbf{n n}: \mathbf{D}|^{2}+\hat{\beta}_{2}|\mathbf{D}|^{2}+\hat{\beta}_{3}|\mathbf{D} \cdot \mathbf{n}|^{2}>0
$$

for any nonzero symmetric traceless matrix $\mathbf{D}$ and unit vector $\mathbf{n}$ if and only if

$$
\hat{\beta}_{2}>0,2 \hat{\beta}_{2}+\hat{\beta}_{3}>0, \frac{3}{2} \hat{\beta}_{2}+\hat{\beta}_{3}+\hat{\beta}_{1}>0
$$

where

$$
\hat{\beta}_{1}=\alpha_{1}+\frac{\gamma_{2}^{2}}{\gamma_{1}}, \hat{\beta}_{2}=\alpha_{4}, \hat{\beta}_{3}=\alpha_{5}+\alpha_{6}-\frac{\gamma_{2}^{2}}{\gamma_{1}} .
$$

Using the above equivalent property we may infer that the energy is dissipated for the EricksenLeslie model derived from the non-inertial Qian-Sheng model. In fact, taking notice of (16) and $s \in(0,1]$, then the coefficients (27) and (28) fulfill the following relations: 


$$
\begin{aligned}
\alpha_{4} & =\mu_{1} \chi^{2}+\beta_{2}^{\prime}-\frac{2}{3} s \beta_{3}^{\prime}>0, \\
2 \alpha_{4}+\alpha_{5}+\alpha_{6}-\frac{\gamma_{2}^{2}}{\gamma_{1}} & =2 \mu_{1} \chi^{2}+2 \beta_{2}^{\prime}-\frac{4}{3} s \beta_{3}^{\prime}+2 s \beta_{3}^{\prime}-2 \mu_{1} \chi^{2} \\
& =2 \beta_{2}^{\prime}+\frac{2}{3} s \beta_{3}^{\prime}>0 \\
\frac{3}{2} \alpha_{4}+\alpha_{5}+\alpha_{6}+\alpha_{1} & =\frac{3}{2} \mu_{1} \chi^{2}+\frac{3}{2} \beta_{2}^{\prime}-s \beta_{3}^{\prime}+2 s \beta_{3}^{\prime}+s^{2} \beta_{1}^{\prime} \\
& =\frac{3}{2} \mu_{1} \chi^{2}+\frac{3}{2} \beta_{2}^{\prime}+s \beta_{3}^{\prime}+s^{2} \beta_{1}^{\prime}>0 .
\end{aligned}
$$

Let us conclude this section by providing the framework of proof for the main result. We first perform the Hilbert expansion for $\left(Q^{\varepsilon}, \mathbf{v}^{\varepsilon}\right)$ :

$$
\begin{aligned}
Q^{\varepsilon}(t, \mathbf{x}) & =Q_{0}(t, \mathbf{x})+\varepsilon Q_{1}(t, \mathbf{x})+\varepsilon^{2} Q_{2}(t, \mathbf{x})+\varepsilon^{3} Q_{3}(t, \mathbf{x})+\varepsilon^{3} Q_{R}(t, \mathbf{x}), \\
\mathbf{v}^{\varepsilon}(t, \mathbf{x}) & =\mathbf{v}_{0}(t, \mathbf{x})+\varepsilon \mathbf{v}_{1}(t, \mathbf{x})+\varepsilon^{2} \mathbf{v}_{2}(t, \mathbf{x})+\varepsilon^{3} \mathbf{v}(t, \mathbf{x}) .
\end{aligned}
$$

Inserting the above expansions into the system (21)-(23) and equating like powers of $\varepsilon$ leads to a hierarchy of equations. We will prove that $\left(Q_{i}, \mathbf{v}_{i}\right)(i=0,1,2)$ and $Q_{3}$ can be determined in this way: $Q_{0}$ must be a critical point of $\mathscr{L}(Q)$, and the system of $\left(Q_{0}, \mathbf{v}_{0}\right)$ can be reduced to the non-inertial Ericksen-Leslie model (see Proposition 3), while $\left(Q_{i}, \mathbf{v}_{i}\right)(i=1,2)$ and $Q_{3}$ solve the linear equations obtained by using the projection operators (see Proposition 4). However, the main difficulty is contained in the analysis of the remainder term $\left(Q_{R}, \mathbf{v}_{R}\right)$. We introduce the definition of good term to derive the system of the remainder. In order to show that the remainder $\left(Q_{R}, \mathbf{v}_{R}\right)$ can be uniformly controlled in $\varepsilon$, we take full advantage of the cancellation relation in the system and the estimates of singular terms (see Lemma 4). We thus obtain the uniform energy estimates for the remainder, see Proposition 5.

\section{Uniaxial Limit for the Non-Inertial Qian-Sheng Model}

By making the Hilbert expansion for the solution of the system (21)-(23), this section is devoted to rigorously justifying the uniaxial limit from the non-inertial Qian-Sheng model to the Ericksen-Leslie model in the framework of smooth solutions.

\subsection{The Hilbert Expansion}

Let $\left(Q^{\varepsilon}, \mathbf{v}^{\varepsilon}\right)$ be a solution of the system (21)-(23), we perform the following Hilbert expansion:

$$
\begin{aligned}
& Q^{\varepsilon}=\sum_{k=0}^{3} \varepsilon^{k} Q_{k}+\varepsilon^{3} Q_{R} \stackrel{\text { def }}{=} \widetilde{Q}+\varepsilon^{3} Q_{R}, \\
& \mathbf{v}^{\varepsilon}=\sum_{k=0}^{2} \varepsilon^{k} \mathbf{v}_{k}+\varepsilon^{3} \mathbf{v}_{R} \stackrel{\text { def }}{=} \tilde{\mathbf{v}}+\varepsilon^{3} \mathbf{v}_{R},
\end{aligned}
$$

where $Q_{i}(0 \leq i \leq 3), \mathbf{v}_{j}(0 \leq j \leq 2)$ are independent of $\varepsilon$, while $\left(Q_{R}, \mathbf{v}_{R}\right)$ are called the remainder term which depend upon $\varepsilon$.

We first introduce the following two definitions

$$
\begin{aligned}
\mathscr{B}\left(Q_{1}, Q_{2}\right) & \stackrel{\text { def }}{=} Q_{1} \cdot Q_{2}+Q_{2}^{T} \cdot Q_{1}^{T}-\frac{2}{3}\left(Q_{1}: Q_{2}\right) \mathbf{I}, \\
\mathscr{C}\left(Q_{1}, Q_{2}, Q_{3}\right) & \stackrel{\text { def }}{=} Q_{1}\left(Q_{2}: Q_{3}\right)+Q_{2}\left(Q_{1}: Q_{3}\right)+Q_{3}\left(Q_{1}: Q_{2}\right) .
\end{aligned}
$$


Let $\widehat{Q}^{\varepsilon}=Q_{1}+\varepsilon Q_{2}+\varepsilon^{2} Q_{3}$, based on the polynomial expansion technique adopted in [5], we obtain the expansion of $\mathscr{T}\left(Q^{\varepsilon}\right)$ in $\varepsilon$ as follows:

$$
\begin{aligned}
\mathscr{T}\left(Q^{\varepsilon}\right)= & \mathscr{T}\left(Q_{0}\right)+\varepsilon \mathscr{H}_{\mathbf{n}}\left(Q_{1}\right)+\varepsilon^{2}\left(\mathscr{H}_{\mathbf{n}}\left(Q_{2}\right)+\mathbf{B}_{1}\right)+\varepsilon^{3}\left(\mathscr{H}_{\mathbf{n}}\left(Q_{3}\right)+\mathbf{B}_{2}\right) \\
& +\varepsilon^{3} \mathscr{H}_{\mathbf{n}}\left(Q_{R}\right)+\varepsilon^{4} \mathscr{T}_{R}^{\varepsilon},
\end{aligned}
$$

where $\mathbf{B}_{1}, \mathbf{B}_{2}$ and $\mathbf{B}^{\varepsilon}$, being all independent of $Q_{R}$, are respectively defined as

$$
\begin{aligned}
& \mathbf{B}_{1}=-\frac{b}{2} \mathscr{B}\left(Q_{1}, Q_{1}\right)+c \mathscr{C}\left(Q_{0}, Q_{1}, Q_{1}\right), \\
& \mathbf{B}_{2}=-b \mathscr{B}\left(Q_{1}, Q_{2}\right)+2 c \mathscr{C}\left(Q_{0}, Q_{1}, Q_{2}\right), \\
& \mathbf{B}^{\varepsilon}=-\frac{b}{2} \sum_{\substack{i+j \geq 4 \\
1 \leq i, j \leq 3}} \varepsilon^{i+j-4} \mathscr{B}\left(Q_{i}, Q_{j}\right)+\frac{c}{3} \sum_{\substack{i+j+k \geq 4 \\
\text { at least two of } i, j, k \text { are not zero }}} \varepsilon^{i+j+k-4} \mathscr{C}\left(Q_{i}, Q_{j}, Q_{k}\right),
\end{aligned}
$$

and the fourth order term $\mathscr{T}_{R}^{\varepsilon}$ in $\varepsilon$ is given by

$$
\begin{aligned}
\mathscr{T}_{R}^{\varepsilon}= & \mathbf{B}^{\varepsilon}-b \mathscr{B}\left(\widehat{Q}^{\varepsilon}, Q_{R}\right)+c \mathscr{C}\left(Q_{R}, \widehat{Q}^{\varepsilon}, Q_{0}\right)+\frac{c}{2} \varepsilon \mathscr{C}\left(Q_{R}, \widehat{Q}^{\varepsilon}, \widehat{Q}^{\varepsilon}\right) \\
& -\frac{b}{2} \varepsilon^{2} \mathscr{B}\left(Q_{R}, Q_{R}\right)+c \varepsilon^{2} \mathscr{C}\left(Q_{R}, Q_{R}, Q_{0}+\varepsilon \widehat{Q}^{\varepsilon}\right)+c \varepsilon^{5} \mathscr{C}\left(Q_{R}, Q_{R}, Q_{R}\right) .
\end{aligned}
$$

For the sake of simplicity, we also denote

$$
\begin{aligned}
& \mathbf{H}_{0}=\mathscr{H}_{\mathbf{n}}\left(Q_{1}\right)+\mathscr{L}\left(Q_{0}\right), \\
& \mathbf{H}_{1}=\mathscr{H}_{\mathbf{n}}\left(Q_{2}\right)+\mathscr{L}\left(Q_{1}\right)+\mathbf{B}_{1}, \\
& \mathbf{H}_{2}=\mathscr{H}_{\mathbf{n}}\left(Q_{3}\right)+\mathscr{L}\left(Q_{2}\right)+\mathbf{B}_{2} .
\end{aligned}
$$

We are now in a position to write down the expansion of the system (21)-(23) and collect the terms (independent of $Q_{R}$ ) with same order of $\varepsilon$. Specifically, we have

- The $O\left(\varepsilon^{-1}\right)$ system

$$
\mathscr{T}\left(Q_{0}\right)=0 .
$$

- The zero-order term in $\varepsilon$

$$
\begin{aligned}
\frac{\partial Q_{0}}{\partial t}+\mathbf{v}_{0} \cdot \nabla Q_{0}= & -\frac{1}{\mu_{1}} \mathbf{H}_{0}+\chi \mathbf{D}_{0}+\mathbf{\Omega}_{0} \cdot Q_{0}-Q_{0} \cdot \mathbf{\Omega}_{0} \\
\frac{\partial \mathbf{v}_{0}}{\partial t}+\mathbf{v}_{0} \cdot \nabla \mathbf{v}_{0}= & -\nabla p_{0}+\nabla \cdot\left(\chi \mathbf{H}_{0}+\beta_{1}^{\prime} Q_{0}\left(Q_{0}: \mathbf{D}_{0}\right)\right. \\
& +\beta_{2}^{\prime} \mathbf{D}_{0}+\beta_{3}^{\prime}\left(Q_{0} \cdot \mathbf{D}_{0}+\mathbf{D}_{0} \cdot Q_{0}\right) \\
& \left.-Q_{0} \cdot \mathbf{H}_{0}+\mathbf{H}_{0} \cdot Q_{0}+\sigma^{d}\left(Q_{0}, Q_{0}\right)\right), \\
\nabla \cdot \mathbf{v}_{0}= & 0 .
\end{aligned}
$$


- The first-order term in $\varepsilon$

$$
\begin{aligned}
\frac{\partial Q_{1}}{\partial t}+\mathbf{v}_{0} \cdot \nabla Q_{1}= & -\frac{1}{\mu_{1}} \mathbf{H}_{1}+\chi \mathbf{D}_{1}+\mathbf{\Omega}_{1} \cdot Q_{0}+\mathbf{\Omega}_{0} \cdot Q_{1} \\
& -Q_{0} \cdot \mathbf{\Omega}_{1}-Q_{1} \cdot \mathbf{\Omega}_{0}-\mathbf{v}_{1} \cdot \nabla Q_{0} \\
\frac{\partial \mathbf{v}_{1}}{\partial t}+\mathbf{v}_{0} \cdot \nabla \mathbf{v}_{1}= & -\mathbf{v}_{1} \cdot \nabla \mathbf{v}_{0}-\nabla p_{1}+\nabla \cdot\left(\chi \mathbf{H}_{1}+\beta_{1}^{\prime}\left(Q_{0}\left(Q_{0}: \mathbf{D}_{1}\right)\right.\right. \\
& \left.+Q_{0}\left(Q_{1}: \mathbf{D}_{0}\right)+Q_{1}\left(Q_{0}: \mathbf{D}_{0}\right)\right)+\beta_{2}^{\prime} \mathbf{D}_{1} \\
& +\beta_{3}^{\prime}\left(Q_{0} \cdot \mathbf{D}_{1}+\mathbf{D}_{0} \cdot Q_{1}+Q_{1} \cdot \mathbf{D}_{0}+\mathbf{D}_{1} \cdot Q_{0}\right) \\
& -Q_{1} \cdot \mathbf{H}_{0}+\mathbf{H}_{0} \cdot Q_{1}-Q_{0} \cdot \mathbf{H}_{1}+\mathbf{H}_{1} \cdot Q_{0} \\
& \left.+\sigma^{d}\left(Q_{1}, Q_{0}\right)+\sigma^{d}\left(Q_{0}, Q_{1}\right)\right) \\
\nabla \cdot \mathbf{v}_{1}= & 0 .
\end{aligned}
$$

- The second-order term in $\varepsilon$

$$
\begin{aligned}
\frac{\partial Q_{2}}{\partial t}+\mathbf{v}_{0} \cdot \nabla Q_{2}= & -\frac{1}{\mu_{1}} \mathbf{H}_{2}+\chi \mathbf{D}_{2}+\mathbf{\Omega}_{2} \cdot Q_{0}+\mathbf{\Omega}_{0} \cdot Q_{2}+\mathbf{\Omega}_{1} \cdot Q_{1} \\
& -Q_{0} \cdot \mathbf{\Omega}_{2}-Q_{2} \cdot \mathbf{\Omega}_{0}-Q_{1} \cdot \mathbf{\Omega}_{1}-\mathbf{v}_{2} \cdot \nabla Q_{0}-\mathbf{v}_{1} \cdot \nabla Q_{1}, \\
\frac{\partial \mathbf{v}_{2}}{\partial t}+\mathbf{v}_{0} \cdot \nabla \mathbf{v}_{2}= & -\mathbf{v}_{2} \cdot \nabla \mathbf{v}_{0}-\mathbf{v}_{1} \cdot \nabla \mathbf{v}_{1}-\nabla p_{2}+\nabla \cdot\left(\chi \mathbf{H}_{2}+\beta_{1}^{\prime}\left(Q_{0}\left(Q_{0}: \mathbf{D}_{2}\right)\right.\right. \\
& +Q_{0}\left(Q_{1}: \mathbf{D}_{1}\right)+Q_{0}\left(Q_{2}: \mathbf{D}_{0}\right)+Q_{1}\left(Q_{0}: \mathbf{D}_{1}\right) \\
& \left.+Q_{1}\left(Q_{1}: \mathbf{D}_{0}\right)+Q_{2}\left(Q_{0}: \mathbf{D}_{0}\right)\right)+\beta_{2}^{\prime} \mathbf{D}_{2} \\
& +\beta_{3}^{\prime}\left(Q_{0} \cdot \mathbf{D}_{2}+\mathbf{D}_{2} \cdot Q_{0}+Q_{1} \cdot \mathbf{D}_{1}+\mathbf{D}_{1} \cdot Q_{1}+Q_{2} \cdot \mathbf{D}_{0}+\mathbf{D}_{0} \cdot Q_{2}\right) \\
& -Q_{2} \cdot \mathbf{H}_{0}+\mathbf{H}_{0} \cdot Q_{2}-Q_{1} \cdot \mathbf{H}_{1}+\mathbf{H}_{1} \cdot Q_{1}-Q_{0} \cdot \mathbf{H}_{2}+\mathbf{H}_{2} \cdot Q_{0} \\
& \left.+\sigma^{d}\left(Q_{2}, Q_{0}\right)+\sigma^{d}\left(Q_{1}, Q_{1}\right)+\sigma^{d}\left(Q_{0}, Q_{2}\right)\right), \\
\nabla \cdot \mathbf{v}_{2}= & 0 .
\end{aligned}
$$

In the sequel, our main task is how to solve $\left(Q_{i}, \mathbf{v}_{i}\right)(0 \leq i \leq 2)$ and $Q_{3}$. First of all, combining the Equation (36) with Proposition 1, we deduce that $Q_{0}$ is a critical point and

$$
Q_{0}(t, \mathbf{x})=s\left(\mathbf{n}(t, \mathbf{x}) \mathbf{n}(t, \mathbf{n})-\frac{1}{3} \mathbf{I}\right),
$$

for some $\mathbf{n}(t, \mathbf{x}) \in \mathbb{S}^{2}$ and $s=\frac{b+\sqrt{b^{2}+24 a c}}{4 c}$.

Proposition 3. Suppose that $\left(Q_{0}, \mathbf{v}_{0}\right)$ is a smooth solution of the system (37)-(39), then ( $\left.\mathbf{n}, \mathbf{v}_{0}\right)$ must be a solution of the Ericksen-Leslie model (1)-(3), where the coefficients are determined by (27)-(29).

Remark 3. Proposition 3 implies that the evolution of $\left(\mathbf{n}, \mathbf{v}_{0}\right)$ can be determined by the system (37)-(39). However, the appearance of unknown $Q_{1}$ in this system leads to the failure of closure. Fortunately, based on Proposition 2, if we project the Equation (37) into the subspace Ker $\mathscr{H}_{\mathbf{n}}$, then $Q_{1}$ vanishs in the Equation (37). Furthermore, if we project the Equation (37) into the subspace $\left(\operatorname{Ker} \mathscr{H}_{\mathbf{n}}\right)^{\perp}$, then we can solve $\mathscr{H}_{\mathbf{n}}\left(Q_{1}\right)$ by using $\left(Q_{0}, \mathbf{v}_{0}\right)$, in other words, $Q_{1}$ can also be removed in the Equation (38).

Proof. This proof is based on the same arguments as Proposition 3.2 in [5]. Recalling the first property $\mathscr{H}_{\mathbf{n}}\left(Q_{1}\right) \in\left(\operatorname{Ker} \mathscr{H}_{\mathbf{n}}\right)^{\perp}$ in Proposition 2, we can deduce from (37) that

$$
\left(\frac{\partial Q_{0}}{\partial t}+\mathbf{v}_{0} \cdot \nabla Q_{0}+Q_{0} \cdot \Omega_{0}-\Omega_{0} \cdot Q_{0}+\frac{1}{\mu_{1}} \mathscr{L}\left(Q_{0}\right)-\chi \mathbf{D}_{0}\right):\left(\mathbf{n n}^{\perp}+\mathbf{n}^{\perp} \mathbf{n}\right)=0 .
$$


Substituting (46) into (47), it follows by a subtle calculation as in [5] that

$$
\mathbf{n}^{\perp} \cdot\left(2 s^{2} \mu_{1} \mathbf{N}-\mathbf{h}-2 \chi \mu_{1} s \mathbf{D}_{0} \cdot \mathbf{n}\right)=0,
$$

which implies

$$
\mathbf{n} \times\left(\mathbf{h}-\gamma_{1} \mathbf{N}-\gamma_{2} \mathbf{D}_{0} \cdot \mathbf{n}\right)=0 .
$$

Applying the definition of the kernel space $\operatorname{Ker} \mathscr{H}_{\mathbf{n}}$ and (46) yields

$$
\frac{\partial Q_{0}}{\partial t}+\mathbf{v}_{0} \cdot \nabla Q_{0}+Q_{0} \cdot \boldsymbol{\Omega}_{0}-\boldsymbol{\Omega}_{0} \cdot Q_{0}=s(\mathbf{n N}+\mathbf{N n}) \in \operatorname{Ker} \mathscr{H}_{\mathbf{n}}
$$

Then from (37) and the definition of the projection operator $\mathscr{P}^{\text {out }}$ we have

$$
\begin{aligned}
\mathscr{H}_{\mathbf{n}}\left(Q_{1}\right)= & \mathscr{P}^{\text {out }}\left(-\mathscr{L}\left(Q_{0}\right)+\mu_{1} \chi \mathbf{D}_{0}\right) \\
= & -\mathscr{L}\left(Q_{0}\right)-\frac{1}{2 s}(\mathbf{h n}+\mathbf{h n})+\frac{1}{s}(\mathbf{h} \cdot \mathbf{n}) \mathbf{n n} \\
& +\mu_{1} \chi \mathbf{D}_{0}-\mu_{1} \chi\left(\mathbf{n n} \cdot \mathbf{D}_{0}+\mathbf{D}_{0} \cdot \mathbf{n n}\right)-2 \mu_{1} \chi\left(\mathbf{D}_{0}: \mathbf{n n}\right) \mathbf{n n},
\end{aligned}
$$

which together with (48) yields

$$
\mathbf{H}_{0}=\mathscr{H}_{\mathbf{n}}\left(Q_{1}\right)+\mathscr{L}\left(Q_{0}\right)=-\mu_{1} s(\mathbf{n} \mathbf{N}+\mathbf{N n})+\mu_{1} \chi \mathbf{D}_{0} .
$$

Consequently, we have

$$
\begin{aligned}
\Sigma^{L} \stackrel{\text { def }}{=} & \chi \mathbf{H}_{0}+\beta_{1}^{\prime} Q_{0}\left(Q_{0}: \mathbf{D}_{0}\right)+\beta_{2}^{\prime} \mathbf{D}_{0}+\beta_{3}^{\prime}\left(Q_{0} \cdot \mathbf{D}_{0}+\mathbf{D}_{0} \cdot Q_{0}\right) \\
& -Q_{0} \cdot \mathbf{H}_{0}+\mathbf{H}_{0} \cdot Q_{0} \\
= & -s \mu_{1} \chi(\mathbf{n N}+\mathbf{N n})+\mu_{1} \chi^{2} \mathbf{D}_{0}+\beta_{1}^{\prime} s^{2}\left(\mathbf{n n}: \mathbf{D}_{0}\right) \mathbf{n n}+\beta_{2}^{\prime} \mathbf{D}_{0} \\
& +\beta_{3}^{\prime} s\left(\mathbf{n n} \cdot \mathbf{D}_{0}+\mathbf{D}_{0} \cdot \mathbf{n n}\right)-\frac{2}{3} \beta_{3}^{\prime} s \mathbf{D}_{0}+\mu_{1} s^{2}(\mathbf{n N}-\mathbf{N n}) \\
& -\mu_{1} s \chi\left(\mathbf{n n} \cdot \mathbf{D}_{0}-\mathbf{D}_{0} \cdot \mathbf{n n}\right)-\frac{1}{3} \beta_{1}^{\prime} s^{2}\left(\mathbf{n n}: \mathbf{D}_{0}\right) \mathbf{I} \\
= & \beta_{1}^{\prime} s^{2}\left(\mathbf{n n}: \mathbf{D}_{0}\right) \mathbf{n n}+\left(-s \chi-s^{2}\right) \mu_{1} \mathbf{N n}+\left(-s \chi+s^{2}\right) \mu_{1} \mathbf{n} \mathbf{N} \\
& +\left(\mu_{1} \chi^{2}+\beta_{2}^{\prime}-\frac{2}{3} \beta_{3}^{\prime} s\right) \mathbf{D}_{0}+\left(\beta_{3}^{\prime} s+\mu_{1} s \chi\right)\left(\mathbf{D}_{0} \cdot \mathbf{n n}\right) \\
& +\left(\beta_{3}^{\prime} s-\mu_{1} s \chi\right)\left(\mathbf{n n} \cdot \mathbf{D}_{0}\right)+\text { pressure terms } \\
= & \sigma^{L}+\text { pressure terms. }
\end{aligned}
$$

From Lemma 3.5 in [5] we know that

$$
\sigma^{E}=\sigma^{d}\left(Q_{0}, Q_{0}\right)
$$

Here $\sigma^{E}$ and $\sigma^{L}$ are the elastic stress and the viscous stress in the Ericksen-Leslie model, respectively. In conclusion, this completes the proof of Proposition 3.

Proposition 4. Let (n, $\left.\mathbf{v}_{0}\right)$ be a smooth solution of the Ericksen-Leslie model (1)-(3) on $[0, T]$ and satisfy

$$
\mathbf{v}_{0} \in C\left([0, T] ; H^{k}\right), \quad \nabla \mathbf{n} \in C\left([0, T] ; H^{k}\right) \quad \text { for } \quad k \geq 20 .
$$


Then there exists the solution $\left(Q_{i}, \mathbf{v}_{i}\right)(i=0,1,2)$ and $Q_{3} \in\left(\operatorname{Ker} \mathscr{H}_{\mathbf{n}}\right)^{\perp}$ of the system (40)-(45) satisfying

$$
\mathbf{v}_{i} \in C\left([0, T] ; H^{k-4 i}\right), \quad Q_{i} \in C\left([0, T] ; H^{k+1-4 i}\right)(i=0,1,2), \quad Q_{3} \in C\left([0, T] ; H^{k-11}\right) .
$$

Proof. The idea of the proof is mainly based on that of [3,5]. To be specific, for $1 \leq i \leq 3$, $Q_{i}$ can be decomposed into two parts: $Q_{i}=Q_{i}^{\top}+Q_{i}^{\perp}$ with $Q_{i}^{\top} \in \operatorname{Ker} \mathscr{H}_{\mathbf{n}}$ and $Q_{i}^{\perp} \in$ $\left(\operatorname{Ker} \mathscr{H}_{\mathbf{n}}\right)^{\perp}$, and then we solve $Q_{i}^{\top}$ and $Q_{i}^{\perp}$ respectively.

Suppose that $\left(\mathbf{n}, \mathbf{v}_{0}\right)$ is a smooth solution of the Ericksen-Leslie model (1)-(3) on $[0, T]$ such that

$$
\mathbf{v}_{0} \in C\left([0, T] ; H^{k}\right), \quad \nabla \mathbf{n} \in C\left([0, T] ; H^{k}\right)
$$

for $k \geq 20$. Thanks to $Q_{0}=s\left(\mathbf{n}(t, \mathbf{x}) \mathbf{n}(t, \mathbf{x})-\frac{1}{3} \mathbf{I}\right)$, we know $Q_{0} \in C\left([0, T], H^{k+1}\right)$. Note that we could solve $Q_{1}^{\perp}$ from (49), and easily get $Q_{1}^{\perp} \in C\left([0, T] ; H^{k-1}\right)$ by Proposition 2 . Thus, the existence of $\left(Q_{1}, \mathbf{v}_{1}\right)$ can be reduced to solving $\left(Q_{1}^{\top}, \mathbf{v}_{1}\right)$

In what follows, we denote by $L\left(Q_{1}^{\top}, \mathbf{v}_{1}\right)$ the terms which only depend on $\left(Q_{1}^{\top}, \mathbf{v}_{1}\right)$ (not their derivatives) linearly with the coefficients belonging to $C\left([0, T] ; H^{k-1}\right)$. We also use $R \in C\left([0, T] ; H^{k-3}\right)$ to denote the terms relying only on $\mathbf{n}, \mathbf{v}_{0}$ and $\mathbf{Q}_{1}^{\perp}$. We denote

$$
\widehat{\mathbf{B}}_{1}(Q, \bar{Q})=-b\left(Q \cdot \bar{Q}-\frac{1}{3}(Q: \bar{Q} \mathbf{I})\right)+c\left(2\left(Q: Q_{0}\right) \bar{Q}+(Q: \bar{Q}) Q_{0}\right) .
$$

Thus we have

$$
\begin{aligned}
\mathbf{B}_{1} & =\widehat{\mathbf{B}}_{1}\left(Q_{1}, Q_{1}\right)=\widehat{\mathbf{B}}_{1}\left(Q_{1}^{\top}, Q_{1}^{\top}\right)+\widehat{\mathbf{B}}_{1}\left(Q_{1}^{\top}, Q_{1}^{\perp}\right)+\widehat{\mathbf{B}}_{1}\left(Q_{1}^{\perp}, Q_{1}^{\top}\right)+\widehat{\mathbf{B}}_{1}\left(Q_{1}^{\perp}, Q_{1}^{\perp}\right) \\
& =\widehat{\mathbf{B}}_{1}\left(Q_{1}^{\top}, Q_{1}^{\top}\right)+L\left(Q_{1}^{\top}, \mathbf{v}_{1}\right) .
\end{aligned}
$$

By a simple calculation we get

$$
\widehat{\mathbf{B}}_{1}\left(Q_{1}^{\top}, Q_{1}^{\top}\right) \in\left(\operatorname{Ker} \mathscr{H}_{\mathbf{n}}\right)^{\perp} .
$$

We are now in a position to derive the system of $\left(Q_{1}^{\top}, \mathbf{v}_{1}\right)$. We denote

$$
\begin{aligned}
& \mathscr{A}_{1}=\mathscr{P}^{\text {in }}\left(\mathscr{L}\left(Q_{1}^{\top}\right)\right), \mathscr{U}_{1}=\mathscr{P}^{\text {in }}\left(\chi \mathbf{D}_{0}+\mathbf{\Omega}_{1} \cdot Q_{0}-Q_{0} \cdot \Omega_{1}\right), \\
& \mathscr{A}_{2}=\mathscr{P}^{\text {out }}\left(\mathscr{L}\left(Q_{1}^{\top}\right)\right), \mathscr{U}_{2}=\mathscr{P}^{\text {out }}\left(\chi \mathbf{D}_{0}+\mathbf{\Omega}_{1} \cdot Q_{0}-Q_{0} \cdot \Omega_{1}\right) .
\end{aligned}
$$

Taking the projection $\mathscr{P}^{i n}$ on both sides of $(40)$, note that $\mathscr{H}_{\mathbf{n}}\left(Q_{2}\right) \in\left(\operatorname{Ker} \mathscr{H}_{\mathbf{n}}\right)^{\perp}$ and $\mathscr{L}\left(Q_{1}\right)=\mathscr{L}\left(Q_{1}^{\top}\right)+R$, from Lemma 1 and (50), there holds

$$
\frac{\partial Q_{1}^{\top}}{\partial t}+\mathbf{v}_{0} \cdot \nabla Q_{1}^{\top}=-\frac{1}{\mu_{1}} \mathscr{A}_{1}+\mathscr{U}_{1}+L\left(Q_{1}^{\top}, \mathbf{v}_{1}\right)+R .
$$

Taking the projection $\mathscr{P}^{\text {out }}$ on both sides of (40) yields

$$
-\frac{1}{\mu_{1}}\left(\mathscr{A}_{2}+\mathscr{H}_{\mathbf{n}}\left(Q_{2}\right)+\widehat{\mathbf{B}}_{1}\left(Q_{1}^{\top}, Q_{1}^{\top}\right)\right)+\mathscr{U}_{2}+L\left(Q_{1}^{\top}, \mathbf{v}_{1}\right)+R=0,
$$

which implies that

$$
\begin{aligned}
\mathbf{H}_{1} & =\mathscr{H}_{\mathbf{n}}\left(Q_{2}\right)+\mathscr{L}\left(Q_{1}\right)+\mathbf{B}_{1} \\
& =\mathscr{A}_{1}+\mu_{1} \mathscr{U}_{2}+L\left(Q_{1}^{\top}, \mathbf{v}_{1}\right)+R .
\end{aligned}
$$


Substituting (52) into (41) and together with (51), we obtain the following closed system for $\left(Q_{1}^{\top}, \mathbf{v}_{1}\right)$

$$
\begin{aligned}
\frac{\partial Q_{1}^{\top}}{\partial t}+\mathbf{v}_{0} \cdot \nabla Q_{1}^{\top}= & -\frac{1}{\mu_{1}} \mathscr{A}_{1}+\mathscr{U}_{1}+L\left(Q_{1}^{\top}, \mathbf{v}_{1}\right)+R, \\
\frac{\partial \mathbf{v}_{1}}{\partial t}+\mathbf{v}_{0} \cdot \nabla \mathbf{v}_{1}= & -\nabla p_{1}+\nabla \cdot\left(\chi\left(\mathscr{A}_{1}+\mu_{1} \mathscr{U}_{2}\right)+\beta_{1}^{\prime} Q_{0}\left(Q_{0}: \mathbf{D}_{1}\right)\right. \\
& +\beta_{2}^{\prime} \mathbf{D}_{1}+\beta_{3}^{\prime}\left(Q_{0} \cdot \mathbf{D}_{1}+\mathbf{D}_{1} \cdot Q_{0}\right) \\
& -Q_{0} \cdot\left(\mathscr{A}_{1}+\mu_{1} \mathscr{U}_{2}\right)+\left(\mathscr{A}_{1}+\mu_{1} \mathscr{U}_{2}\right) \cdot Q_{0} \\
& \left.+\sigma^{d}\left(Q_{1}^{\top}, Q_{0}\right)+\sigma^{d}\left(Q_{0}, Q_{1}^{\top}\right)+L\left(Q_{1}^{\top}, \mathbf{v}_{1}\right)+R\right), \\
\nabla \cdot \mathbf{v}_{1}= & 0 .
\end{aligned}
$$

Clearly, (53)-(55) is a linear system with respect to $\left(Q_{1}^{\top}, \mathbf{v}_{1}\right)$. The solvability of this system strongly relies on the dissipation relations. For this reason, we introduce the energy functional

$$
\mathscr{E}(t) \stackrel{\text { def }}{=} \frac{\beta_{2}^{\prime}}{3}\left\langle\mathbf{v}_{1}, \mathbf{v}_{1}\right\rangle+\left\langle Q_{1}^{\top}, \mathscr{L}\left(Q_{1}^{\top}\right)\right\rangle+\left\langle Q_{1}^{\top}, Q_{1}^{\top}\right\rangle
$$

Bearing in mind relations between the coefficients in (30), it follows that

$$
\begin{aligned}
- & \left\langle\beta_{1}^{\prime} Q_{0}\left(Q_{0}: \mathbf{D}_{1}\right)+\beta_{2}^{\prime} \mathbf{D}_{1}+\beta_{3}^{\prime}\left(Q_{0} \cdot \mathbf{D}_{1}+\mathbf{D}_{1} \cdot Q_{0}\right), \nabla \mathbf{v}_{1}\right\rangle \\
& =-s^{2} \beta_{1}^{\prime}\left\|\mathbf{n n}: \mathbf{D}_{1}\right\|_{L^{2}}^{2}-\left(\beta_{2}^{\prime}-\frac{2}{3} s \beta_{3}^{\prime}\right)\left\|\mathbf{D}_{1}\right\|_{L^{2}}^{2}-2 s \beta_{3}^{\prime}\left\|\mathbf{n} \cdot \mathbf{D}_{1}\right\|_{L^{2}}^{2} \\
& \leq 0
\end{aligned}
$$

Meanwhile, taking into account the following key dissipation

$$
\begin{aligned}
- & \left\langle\chi\left(\mathscr{A}_{1}+\mu_{1} \mathscr{U}_{2}\right)-Q_{0} \cdot\left(\mathscr{A}_{1}+\mu_{1} \mathscr{U}_{2}\right)+\left(\mathscr{A}_{1}+\mu_{1} \mathscr{U}_{2}\right) \cdot Q_{0}, \nabla \mathbf{v}_{1}\right\rangle+\left\langle\mathscr{U}_{1}, \mathscr{L}\left(Q_{1}^{\top}\right)\right\rangle \\
= & -\left\langle\mathscr{A}_{1}+\mu_{1} \mathscr{U}_{2}, \chi \mathbf{D}_{0}+\mathbf{\Omega}_{1} \cdot Q_{0}-Q_{0} \cdot \mathbf{\Omega}_{1}\right\rangle+\left\langle\mathscr{U}_{1}, \mathscr{L}\left(Q_{1}^{\top}\right)\right\rangle \\
= & -\left\langle\mathscr{P}^{\text {in }}\left(\mathscr{L}\left(Q_{1}^{\top}\right)\right), \chi \mathbf{D}_{0}+\mathbf{\Omega}_{1} \cdot Q_{0}-Q_{0} \cdot \mathbf{\Omega}_{1}\right\rangle \\
& +\left\langle\mathscr{P}^{\text {in }}\left(\chi \mathbf{D}_{0}+\mathbf{\Omega}_{1} \cdot Q_{0}-Q_{0} \cdot \boldsymbol{\Omega}_{1}\right), \mathscr{L}\left(Q_{1}^{\top}\right)\right\rangle \\
& -\mu_{1}\left\langle\mathscr{U}_{2}, \chi \mathbf{D}_{0}+\mathbf{\Omega}_{1} \cdot Q_{0}-Q_{0} \cdot \mathbf{\Omega}_{1}\right\rangle \\
= & -\mu_{1}\left\langle\mathscr{P}^{\text {out }}\left(\chi \mathbf{D}_{0}+\mathbf{\Omega}_{1} \cdot Q_{0}-Q_{0} \cdot \mathbf{\Omega}_{1}\right), \chi \mathbf{D}_{0}+\mathbf{\Omega}_{1} \cdot Q_{0}-Q_{0} \cdot \mathbf{\Omega}_{1}\right\rangle \\
\leq & 0 .
\end{aligned}
$$

Then we can deduce by a simple energy estimate that there exists a positive constant $C$ such that

$$
\frac{d}{d t} \mathscr{E}(t) \leq C(1+\mathscr{E}(t))
$$

The similar method gives rise to the estimate of higher-order derivative for $\left(Q_{1}^{\top}, \mathbf{v}_{1}\right)$. Hence, this indicates the existence of $\left(Q_{1}, \mathbf{v}_{1}\right)$.

Finally, from (52) we can solve $Q_{2}^{\perp}$ as

$$
Q_{2}^{\perp}=\mathscr{H}_{\mathbf{n}}^{-1}\left(-\mathscr{L}\left(Q_{1}\right)-\mathbf{B}_{1}+\mathscr{A}_{1}+\mu_{1} \mathscr{U}_{2}+L\left(Q_{1}^{\top}, \mathbf{v}_{1}\right)+R\right) \in C\left([0, T] ; H^{k-5}\right) .
$$


Then $\left(Q_{2}^{\top}, \mathbf{v}_{2}\right)$ can be solved in a similar way as $\left(Q_{1}^{\top}, \mathbf{v}_{1}\right) . Q_{3}$ can be solved as in (56) (unique up to a term in $\operatorname{Ker} \mathscr{H}_{\mathbf{n}}$ ). Here we omit the details.

Lemma 1. It holds that

$$
\begin{aligned}
& \mathscr{P}^{\text {out }}\left(\frac{\partial Q_{1}}{\partial t}+\mathbf{v}_{0} \cdot \nabla Q_{1}\right)=L\left(Q_{1}^{\top}\right)+R, \\
& \mathscr{P}^{\text {in }}\left(\frac{\partial Q_{1}}{\partial t}+\mathbf{v}_{0} \cdot \nabla Q_{1}\right)=\frac{\partial Q_{1}^{\top}}{\partial t}+\mathbf{v}_{0} \cdot \nabla Q_{1}^{\top}+L\left(Q_{1}^{\top}\right)+R .
\end{aligned}
$$

Proof. For further details, one refers to [5].

\subsection{The System for the Remainder}

This subsection aims to deriving the remainder system and uniform estimates for the remainder. The previous Proposition 4 tells us that $\mathbf{v}_{i} \in C\left([0, T] ; H^{k-4 i}\right)$ for $i=0,1,2$ and $Q_{i} \in C\left([0, T] ; H^{k+1-4 i}\right)$ for $i=0,1,2,3$. Hence, $\mathbf{v}_{i}$ and $Q_{i}$ will be treated as known functions in this subsection. In what follows, we denote by $C$ a constant depending on $\sum_{i=0}^{2} \sup _{t \in[0, T]}\left\|\mathbf{v}_{i}(t)\right\|_{H^{k-4 i}}$ and $\sum_{i=0}^{3} \sup _{t \in[0, T]}\left\|Q_{i}(t)\right\|_{H^{k+1-4 i}}$, and independent of $\varepsilon$.

By the Hilbert expansion (32) and (33), we have

$$
Q_{R}=\frac{1}{\mathcal{E}^{3}}\left(Q^{\varepsilon}-\widetilde{Q}\right), \quad \mathbf{v}_{R}=\frac{1}{\varepsilon^{3}}\left(\mathbf{v}^{\varepsilon}-\tilde{\mathbf{v}}\right),
$$

where $Q_{R}$ and $\mathbf{v}_{R}$ depend on $\varepsilon$. For the sake of deriving the evolution equations of the remainder $\left(Q_{R}, \mathbf{v}_{R}\right)$, we rewrite the system of $\left(Q^{\varepsilon}, \mathbf{v}^{\varepsilon}\right)$ as follows:

$$
\begin{aligned}
\frac{\partial}{\partial t} Q^{\varepsilon} & =\mathcal{M}\left(Q^{\varepsilon}\right)+\mathcal{G}\left(Q^{\varepsilon}, \mathbf{v}^{\varepsilon}\right), \\
\frac{\partial}{\partial t} \mathbf{v}^{\varepsilon} & =\mathscr{P}_{\operatorname{div}} \nabla \cdot\left(\mathcal{K}\left(Q^{\varepsilon}\right)+\mathcal{S}\left(Q^{\varepsilon}, \mathbf{v}^{\varepsilon}\right)\right) .
\end{aligned}
$$

where $\mathscr{P}_{\text {div }}$ is a projection operator mapping a vector field into its solenoidal part, $\mathcal{M}\left(Q^{\varepsilon}\right)=\frac{1}{\mu_{1}} \mathbf{H}\left(Q^{\varepsilon}\right)$ and

$$
\begin{aligned}
\mathcal{G}\left(Q^{\varepsilon}, \mathbf{v}^{\varepsilon}\right) & =\chi \mathbf{D}^{\varepsilon}+\mathbf{\Omega}^{\varepsilon} \cdot Q^{\varepsilon}-Q^{\varepsilon} \cdot \mathbf{\Omega}^{\varepsilon}-\mathbf{v}^{\varepsilon} \cdot \nabla Q^{\varepsilon} \\
\mathcal{K}\left(Q^{\varepsilon}\right) & =\chi \mathbf{H}\left(Q^{\varepsilon}\right)-Q^{\varepsilon} \cdot \mathbf{H}\left(Q^{\varepsilon}\right)+\mathbf{H}\left(Q^{\varepsilon}\right) \cdot Q^{\varepsilon} \\
\mathcal{S}\left(Q^{\varepsilon}, \mathbf{v}^{\varepsilon}\right) & =\beta_{1}^{\prime} Q^{\varepsilon}\left(Q^{\varepsilon}: \mathbf{D}^{\varepsilon}\right)+\beta_{2}^{\prime} \mathbf{D}^{\varepsilon}+\beta_{3}^{\prime}\left(Q^{\varepsilon} \cdot \mathbf{D}^{\varepsilon}+\mathbf{D}^{\varepsilon} \cdot Q^{\varepsilon}\right)+\sigma^{d}\left(Q^{\varepsilon}, Q^{\varepsilon}\right)-\mathbf{v}^{\varepsilon} \otimes \mathbf{v}^{\varepsilon} \\
& \triangleq \mathcal{S}_{1}\left(Q^{\varepsilon}, \mathbf{v}^{\varepsilon}\right)+\beta_{2}^{\prime} \mathbf{D}^{\varepsilon}+\mathcal{S}_{2}\left(Q^{\varepsilon}, \mathbf{v}^{\varepsilon}\right)+\mathcal{S}_{3}\left(Q^{\varepsilon}\right)+\mathcal{S}_{4}\left(\mathbf{v}^{\varepsilon}\right) .
\end{aligned}
$$

Consequently, from (57) we can get

$$
\begin{aligned}
\frac{\partial}{\partial t} Q_{R}= & \frac{1}{\mathcal{E}^{3}}\left(\mathcal{M}\left(Q^{\varepsilon}\right)-\mathcal{M}(\widetilde{Q})\right)+\frac{1}{\varepsilon^{3}}\left(\mathcal{G}\left(Q^{\varepsilon}, \mathbf{v}^{\varepsilon}\right)-\mathcal{G}(\widetilde{Q}, \tilde{\mathbf{v}})\right) \\
& +\frac{1}{\varepsilon^{3}}\left(\mathcal{M}(\widetilde{Q})+\mathcal{G}(\widetilde{Q}, \tilde{\mathbf{v}})-\frac{\partial}{\partial t} \widetilde{Q}\right), \\
\frac{\partial}{\partial t} \mathbf{v}_{R}= & \mathscr{P}_{\operatorname{div}} \nabla \cdot\left(\frac{1}{\mathcal{E}^{3}}\left(\mathcal{K}\left(Q^{\varepsilon}\right)-\mathcal{K}(\widetilde{Q})\right)+\frac{1}{\mathcal{E}^{3}}\left(\mathcal{S}\left(Q^{\varepsilon}, \mathbf{v}^{\varepsilon}\right)-\mathcal{S}(\widetilde{Q}, \tilde{\mathbf{v}})\right)\right) \\
& +\frac{1}{\varepsilon^{3}}\left(\mathscr{P}_{\operatorname{div}} \nabla \cdot(\mathcal{K}(\widetilde{Q})+\mathcal{S}(\widetilde{Q}, \tilde{\mathbf{v}}))-\frac{\partial}{\partial t} \tilde{\mathbf{v}}\right) .
\end{aligned}
$$

Needless to say, this is a tedious task if we want to precisely express the right-hand terms of the above system. To greatly simplifying the derivation of the remainder system, 
as will be shown later, we introduce a notation $\Re$, called good terms, to stand for all terms in (60) and (61) satisfying

$$
\|\mathfrak{R}\|_{L^{2}}+\varepsilon\|\nabla \mathfrak{R}\|_{L^{2}}+\varepsilon^{2}\|\Delta \mathfrak{R}\|_{L^{2}} \leq C(\varepsilon E)(1+E+\varepsilon F)+\varepsilon f(E),
$$

where $\mathfrak{R}$ possibly depends on $\left\|Q_{i}\right\|$ and parameters of the system but is independent of $\varepsilon, C(\cdot)$ and $f(\cdot): \mathbb{R}^{+} \cup\{0\} \mapsto \mathbb{R}^{+} \cup\{0\}$ are increasing functions, $E$ and $F$ are respectively defined as

$$
\begin{aligned}
& E=\left\|Q_{R}\right\|_{H^{1}}+\varepsilon\left\|\Delta Q_{R}\right\|_{L^{2}}+\varepsilon^{2}\left\|\nabla \Delta Q_{R}\right\|_{L^{2}}+\left\|\mathbf{v}_{R}\right\|_{L^{2}}+\varepsilon\left\|\nabla \mathbf{v}_{R}\right\|_{L^{2}}+\varepsilon^{2}\left\|\Delta \mathbf{v}_{R}\right\|_{L^{2}}, \\
& F=\varepsilon\left\|\nabla \mathscr{L}\left(Q_{R}\right)\right\|_{L^{2}}+\varepsilon^{2}\left\|\Delta \mathscr{L}\left(Q_{R}\right)\right\|_{L^{2}}+\varepsilon^{2}\left\|\Delta \nabla \mathbf{v}_{R}\right\|_{L^{2}} .
\end{aligned}
$$

It needs to mention that in order to get the property (62) of good terms $\Re$, we take full advantage of the following simple facts obtained by Sobolev embedding theorem that for $k=0,1,2$ and some constant $C$, there holds

$$
\begin{aligned}
& \varepsilon^{k}\left\|Q_{R}\right\|_{H^{k}}+\varepsilon^{k}\left\|\mathbf{v}_{R}\right\|_{H^{k}} \leq E, \quad \varepsilon\left\|Q_{R}\right\|_{L^{\infty}}+\varepsilon^{2}\left\|\mathbf{v}_{R}\right\|_{L^{\infty}} \leq C E, \\
& \varepsilon^{k+1}\left\|\mathcal{L}\left(Q_{R}\right)\right\|_{H^{k}}+\varepsilon^{3}\left\|\nabla \mathbf{v}_{R}\right\|_{L^{\infty}} \leq C(E+\varepsilon F) \\
& \left\|Q^{\varepsilon}\right\|_{H^{k}} \leq C(\varepsilon E), \quad\left\|\mathbf{v}^{\varepsilon}\right\|_{H^{k}} \leq C(\varepsilon E) .
\end{aligned}
$$

The unique advantage of the good terms $\mathfrak{R}$ lies in the right-hand side being controlled by $C(1+E)$ as $\varepsilon \rightarrow 0$. This will contribute to a uniformly closed energy estimate in $\varepsilon$, see Proposition 5 for details.

With the definition of good term $\mathfrak{R}$, we could now analyze the right-hand terms of the system (60) and (61). First, by means of the choices of $Q_{i}(0 \leq i \leq 3), \mathbf{v}_{j}(0 \leq j \leq 2)$, it is easy to see that

$$
\left\|\frac{1}{\mathcal{E}^{3}}\left(\mathcal{K}(\widetilde{Q})+\mathcal{G}(\widetilde{Q}, \tilde{\mathbf{v}})-\frac{\partial}{\partial t} \widetilde{Q}\right)\right\|_{H^{2^{\prime}}} \quad\left\|\frac{1}{\mathcal{E}^{3}}\left(\mathscr{P}_{\operatorname{div}} \nabla \cdot(\mathcal{M}(\widetilde{Q})+\mathcal{S}(\widetilde{Q}, \tilde{\mathbf{v}}))-\frac{\partial}{\partial t} \tilde{\mathbf{v}}\right)\right\|_{H^{2}}
$$

can be all controlled by a constant uniformly in $\varepsilon$, thus can be absorbed in $\mathfrak{R}$.

In addition, for the remaining terms, we have the following two lemmas:

Lemma 2. For the terms of $\mathcal{G}$ and $\mathcal{S}$, it follows that

$$
\begin{aligned}
\mathcal{G}\left(Q^{\varepsilon}, \mathbf{v}^{\varepsilon}\right)-\mathcal{G}(\widetilde{Q}, \tilde{\mathbf{v}}) & =\varepsilon^{3}\left(\chi \mathbf{D}_{R}+\mathbf{\Omega}_{R} \cdot Q_{0}-Q_{0} \cdot \mathbf{\Omega}_{R}\right)+\varepsilon^{3} \mathfrak{R}, \\
\mathcal{S}_{1}\left(Q^{\varepsilon}, \mathbf{v}^{\varepsilon}\right)-\mathcal{S}_{1}(\widetilde{Q}, \tilde{\mathbf{v}}) & =\varepsilon^{3} \beta_{1}^{\prime} Q_{0}\left(Q_{0}: \mathbf{D}_{R}\right)+\varepsilon^{3} \mathfrak{R}, \\
\mathcal{S}_{2}\left(Q^{\varepsilon}, \mathbf{v}^{\varepsilon}\right)-\mathcal{S}_{2}(\widetilde{Q}, \tilde{\mathbf{v}}) & =\varepsilon^{3} \beta_{3}^{\prime}\left(Q_{0} \cdot \mathbf{D}_{R}+\mathbf{D}_{R} \cdot Q_{0}\right)+\varepsilon^{3} \mathfrak{R}, \\
\mathcal{S}_{3}\left(Q^{\varepsilon}\right)-\mathcal{S}_{3}(\widetilde{Q}) & =\varepsilon^{3} \mathfrak{R}, \\
\mathcal{S}_{4}\left(\mathbf{v}^{\varepsilon}\right)-\mathcal{S}_{4}(\tilde{\mathbf{v}}) & =\varepsilon^{3} \mathfrak{R} .
\end{aligned}
$$

Proof. First, for $0 \leq k \leq 2$, noting that

$$
\begin{aligned}
\varepsilon^{k+3}\left\|\mathbf{\Omega}^{\varepsilon} \cdot Q_{R}\right\|_{H^{k}} & \leq \varepsilon^{3} C\left(1+\varepsilon^{3}\left\|\nabla \mathbf{v}_{R}\right\|_{L^{\infty}}\right)\left\|\varepsilon^{k} Q_{R}\right\|_{H^{k}} \\
& \leq \varepsilon^{3} C(1+\varepsilon F) E, \\
\varepsilon^{k}\left\|\mathbf{v}^{\varepsilon} \cdot \nabla Q^{\varepsilon}-\tilde{\mathbf{v}} \cdot \nabla \widetilde{Q}\right\|_{H^{k}} & \leq \varepsilon^{k}\left\|\mathbf{v}^{\varepsilon} \cdot \nabla Q^{\varepsilon}-\mathbf{v}^{\varepsilon} \cdot \nabla \widetilde{Q}\right\|_{H^{k}}+\varepsilon^{k}\left\|\mathbf{v}^{\varepsilon} \cdot \nabla \widetilde{Q}-\tilde{\mathbf{v}} \cdot \nabla \widetilde{Q}\right\|_{H^{k}} \\
& \leq \varepsilon^{3}\left\|\varepsilon^{k} Q_{R}\right\|_{H^{k}}\left\|\mathbf{v}^{\varepsilon}\right\|_{L^{\infty}}+\varepsilon^{3}\left\|\varepsilon^{k} \mathbf{v}_{R}\right\|_{H^{k}}\|\nabla \widetilde{Q}\|_{L^{\infty}} \\
& \leq \varepsilon^{3} C(1+\varepsilon E) E,
\end{aligned}
$$


then we have

$$
\begin{aligned}
\boldsymbol{\Omega}^{\varepsilon} \cdot & Q^{\varepsilon}-Q^{\varepsilon} \cdot \mathbf{\Omega}^{\varepsilon}-\widetilde{\mathbf{\Omega}} \cdot \widetilde{Q}+\widetilde{Q} \cdot \widetilde{\mathbf{\Omega}}+\mathbf{v}^{\varepsilon} \cdot \nabla Q^{\varepsilon}-\tilde{\mathbf{v}} \cdot \nabla \widetilde{Q} \\
& =\varepsilon^{3}\left(\boldsymbol{\Omega}^{\varepsilon} \cdot Q_{R}-Q_{R} \cdot \mathbf{\Omega}^{\varepsilon}\right)+\varepsilon^{3}\left(\boldsymbol{\Omega}_{R} \cdot \widetilde{Q}-\widetilde{Q} \cdot \boldsymbol{\Omega}_{R}\right)+\varepsilon^{3} \mathfrak{R} \\
& =\varepsilon^{3}\left(\boldsymbol{\Omega}_{R} \cdot Q_{0}-Q_{0} \cdot \boldsymbol{\Omega}_{R}\right)+\varepsilon^{3} \mathfrak{R} .
\end{aligned}
$$

Therefore, (67) holds.

For (70), we have

$$
\begin{aligned}
\varepsilon^{k}\left\|\sigma^{d}\left(Q^{\varepsilon}, Q^{\varepsilon}\right)-\sigma^{d}(\widetilde{Q}, \widetilde{Q})\right\|_{H^{k}} & =\varepsilon^{3+k}\left\|\sigma^{d}\left(Q^{\varepsilon}, Q_{R}\right)+\sigma^{d}\left(Q_{R}, \widetilde{Q}\right)\right\|_{H^{k}} \\
& \leq C \varepsilon^{3}\left\|\varepsilon^{k} \nabla Q_{R}\right\|_{H^{k}}\left(1+\left\|\varepsilon^{3} \nabla Q_{R}\right\|_{L^{\infty}}\right) \\
& \leq \varepsilon^{3} C(1+\varepsilon E) E .
\end{aligned}
$$

Finally, (68), (69) and (71) can be deduced in the same way.

Lemma 3. For the terms of $\mathcal{M}$ and $\mathcal{K}$, it follows that

$$
\begin{aligned}
\mathcal{M}\left(Q^{\varepsilon}\right)-\mathcal{M}(\widetilde{Q}) & =\frac{\varepsilon^{2}}{\mu_{1}} \mathscr{H}_{\mathbf{n}}^{\varepsilon}\left(Q_{R}\right)+\varepsilon^{3} \Re \\
\mathcal{K}\left(Q^{\varepsilon}\right)-\mathcal{K}(\widetilde{Q}) & =\varepsilon^{2}\left(\chi \mathscr{H}_{\mathbf{n}}^{\varepsilon}\left(Q_{R}\right)-Q_{0} \cdot \mathscr{H}_{\mathbf{n}}^{\varepsilon}\left(Q_{R}\right)+\mathscr{H}_{\mathbf{n}}^{\varepsilon}\left(Q_{R}\right) \cdot Q_{0}\right)+\varepsilon^{3} \Re .
\end{aligned}
$$

Proof. By (34) and the definitions of $\mathbf{H}_{i}(i=0,1,2)$, then $\mu_{1} \mathcal{M}\left(Q^{\varepsilon}\right)$ can be expanded as

$$
\mu_{1} \mathcal{M}\left(Q^{\varepsilon}\right)=\mathbf{H}_{0}+\varepsilon \mathbf{H}_{1}+\varepsilon^{2} \mathbf{H}_{2}+\varepsilon^{2} \mathbf{H}_{R}+\varepsilon^{3} \mathscr{T}_{R},
$$

where $\mathbf{H}_{R}=\mathscr{H}_{\mathbf{n}}^{\varepsilon}\left(Q_{R}\right) \stackrel{\text { def }}{=} \mathscr{H}_{\mathbf{n}}\left(Q_{R}\right)+\varepsilon \mathscr{L}\left(Q_{R}\right)$. Notice that, for $0 \leq k \leq 2$,

$$
\varepsilon^{k}\left\|\mathscr{T}_{R}\right\|_{H^{k}} \leq C(\varepsilon E)(1+E+\varepsilon F)+\varepsilon f(E),
$$

which can be also absorbed in $\Re$. So we have

$$
\mathcal{M}\left(Q^{\varepsilon}\right)-\mathcal{M}(\widetilde{Q})=\frac{\varepsilon^{2}}{\mu_{1}} \mathscr{H}_{\mathbf{n}}^{\varepsilon}\left(Q_{R}\right)+\varepsilon^{3} \Re .
$$

It is easy to obtain (73) from (72).

In conclusion, combining Lemma 2 with Lemma 3 leads to the following remainder system:

$$
\begin{aligned}
\frac{\partial Q_{R}}{\partial t}= & -\frac{1}{\mu_{1} \varepsilon} \mathscr{H}_{\mathbf{n}}^{\varepsilon}\left(Q_{R}\right)+\chi \mathbf{D}_{R}+\mathbf{\Omega}_{R} \cdot Q_{0}-Q_{0} \cdot \boldsymbol{\Omega}_{R}+\mathfrak{R}, \\
\frac{\partial \mathbf{v}_{R}}{\partial t}= & -\nabla p_{R}+\frac{1}{\varepsilon} \nabla \cdot\left(\chi \mathscr{H}_{\mathbf{n}}^{\varepsilon}\left(Q_{R}\right)-Q_{0} \cdot \mathscr{H}_{\mathbf{n}}^{\varepsilon}\left(Q_{R}\right)+\mathscr{H}_{\mathbf{n}}^{\varepsilon}\left(Q_{R}\right) \cdot Q_{0}\right) \\
& +\nabla \cdot\left(\beta_{1}^{\prime} Q_{0}\left(Q_{0}: \mathbf{D}_{R}\right)+\beta_{2}^{\prime} \mathbf{D}_{R}+\beta_{3}^{\prime}\left(Q_{0} \cdot \mathbf{D}_{R}+\mathbf{D}_{R} \cdot Q_{0}\right)\right)+\nabla \cdot \mathfrak{R}+\mathfrak{R}, \\
\nabla \cdot \mathbf{v}_{R}= & 0 .
\end{aligned}
$$

It can be observed that the remainder system (74)-(76) involves the singular term $\frac{1}{\varepsilon} \mathscr{H}_{\mathbf{n}}^{\varepsilon}\left(Q_{R}\right)$ in $\varepsilon$. Therefore, as shown in [3,5], in order to obtain the uniform energy estimates, we have to construct the following suitable energy functionals 


$$
\begin{aligned}
\mathfrak{E}(t)= & \frac{1}{2} \int_{\mathbb{R}^{3}}\left(\left|\mathbf{v}_{R}\right|^{2}+\left|Q_{R}\right|^{2}+\frac{1}{\varepsilon} \mathscr{H}_{\mathbf{n}}^{\varepsilon}\left(Q_{R}\right): Q_{R}\right) \\
& +\varepsilon^{2}\left(\left|\nabla \mathbf{v}_{R}\right|^{2}+\frac{1}{\varepsilon} \mathscr{H}_{\mathbf{n}}^{\varepsilon}\left(\partial_{i} Q_{R}\right): \partial_{i} Q_{R}\right) \\
& +\varepsilon^{4}\left(\left|\Delta \mathbf{v}_{R}\right|^{2}+\frac{1}{\varepsilon} \mathscr{H}_{\mathbf{n}}^{\varepsilon}\left(\Delta Q_{R}\right): \Delta Q_{R}\right) d \mathbf{x} \\
\mathfrak{F}(t)= & \int_{\mathbb{R}^{3}}\left(v\left|\nabla \mathbf{v}_{R}\right|^{2}+\frac{1}{\mu_{1} \varepsilon^{2}} \mathscr{H}_{\mathbf{n}}^{\varepsilon}\left(Q_{R}\right): \mathscr{H}_{\mathbf{n}}^{\varepsilon}\left(Q_{R}\right)\right) \\
& +\varepsilon^{2}\left(v\left|\Delta \mathbf{v}_{R}\right|^{2}+\frac{1}{\mu_{1} \varepsilon^{2}} \mathscr{H}_{\mathbf{n}}^{\varepsilon}\left(\partial_{i} Q_{R}\right): \mathscr{H}_{\mathbf{n}}^{\varepsilon}\left(\partial_{i} Q_{R}\right)\right) \\
& +\varepsilon^{4}\left(v\left|\nabla \Delta \mathbf{v}_{R}\right|^{2}+\frac{1}{\mu_{1} \varepsilon^{2}} \mathscr{H}_{\mathbf{n}}^{\varepsilon}\left(\Delta Q_{R}\right): \mathscr{H}_{\mathbf{n}}^{\varepsilon}\left(\Delta Q_{R}\right)\right) d \mathbf{x},
\end{aligned}
$$

where $v=\frac{1}{3} \beta_{2}^{\prime}>0$ and $\mathscr{H}_{\mathbf{n}}^{\mathcal{E}}(Q)=\mathscr{H}_{\mathbf{n}}(Q)+\varepsilon \mathscr{L}(Q)$.

The a priori estimate for the remainder $\left(Q_{R}, \mathbf{v}_{R}\right)$ is stated as follows.

Proposition 5. There exist two functions $C$ and $f$ depending on $Q_{i}, \mathbf{v}_{j}$ and the parameters of the system (but independent of $\varepsilon$ ), such that if $\left(Q_{R}, \mathbf{v}_{R}\right)$ be a smooth solution of the system (74)-(76) on $[0, T]$, then for any $t \in[0, T]$, it holds that

$$
\frac{d}{d t} \mathfrak{E}(t)+\mathfrak{F}(t) \leq C(\varepsilon \mathfrak{E})(1+\mathfrak{E})+\varepsilon f(\mathfrak{E})+C(\varepsilon \mathfrak{E}) \mathcal{E} \mathfrak{F} .
$$

The proof of Proposition 5 will be presented in next subsection. Provided that Proposition 5 holds, we can now adopt a standard argument to finish the proof of the main result in this article.

Proof of Theorem 1. Given the initial data $\left(\mathbf{v}_{0}^{\varepsilon}, Q_{0}^{\varepsilon}\right) \in H^{2} \times H^{3}$, it can be proved from the standard energy method in $[23,24,29,31]$ that there exists a maximal time $T_{\varepsilon}>0$ and a unique solution $\left(\mathbf{v}^{\varepsilon}, Q^{\varepsilon}\right)$ of the system (21)-(23) such that

$$
Q^{\varepsilon} \in C\left(\left[0, T_{\varepsilon}\right) ; H^{3}\right) \cap L^{2}\left(0, T_{\varepsilon} ; H^{4}\right), \quad \mathbf{v}^{\varepsilon} \in C\left(\left[0, T_{\varepsilon}\right) ; H^{2}\right) \cap L^{2}\left(0, T_{\varepsilon} ; H^{3}\right) .
$$

From Proposition 5 we have

$$
\frac{d}{d t} \mathfrak{E}(t)+\mathfrak{F}(t) \leq C(\varepsilon \mathfrak{E})(1+\mathfrak{E})+\varepsilon f(\mathfrak{E})+C(\varepsilon \mathfrak{E}) \mathfrak{E} \mathfrak{F} .
$$

for any $t \in\left[0, T_{\varepsilon}\right]$. Under the assumptions of Theorem 1 , it follows that

$$
\mathfrak{E}(0) \leq C_{1}\left(\left\|\mathbf{v}_{I, R}^{\varepsilon}\right\|_{H^{2}}+\left\|Q_{I, R}^{\varepsilon}\right\|_{H^{3}}+\varepsilon^{-1}\left\|\mathscr{P}^{\text {out }}\left(Q_{I, R}^{\varepsilon}\right)\right\|_{L^{2}}\right) \leq C_{1} E_{0} .
$$

Let $E_{1}=\left(2+C_{1} E_{0}\right) e^{T}-2>\mathfrak{E}(0)$, and

$$
T_{1}=\sup \left\{t \in\left[0, T_{\varepsilon}\right]: \mathfrak{E}(t) \leq E_{1}\right\} .
$$

If we take $\varepsilon_{0}$ small enough such that

$$
C\left(\varepsilon_{0} E_{1}\right) \leq 1, \quad \varepsilon_{0} f\left(E_{1}\right) \leq 1, \quad \varepsilon_{0} \leq 1 / 2,
$$

then for $t \leq T_{1}$, there holds

$$
\frac{d}{d t} \mathfrak{E}(t) \leq 2+\mathfrak{E} .
$$

Therefore, we can infer by means of a continuous argument that $T \leq T_{\mathcal{E}}$ and $\mathfrak{E}(t) \leq E_{1}$ for $t \in[0, T]$. This completes the proof of Theorem 1 . 


\subsection{Proof of Proposition 5}

To finish the proof of Proposition 5, we also need to control two singular terms $\frac{1}{\varepsilon}\left\langle\partial_{t}(\mathbf{n n}) \cdot Q, Q\right\rangle$ and $\frac{1}{\varepsilon}\left\langle Q: \partial_{t}(\mathbf{n n}), Q: \mathbf{n n}\right\rangle$ which come from $\frac{1}{\varepsilon} \frac{d}{d t}\left\langle Q_{R}, \mathscr{H}_{\mathbf{n}}^{\varepsilon}\left(Q_{R}\right)\right\rangle$. Fortunately, two singular terms are all bounded as demonstrated in the following Lemma 4.

Lemma 4. For any $\delta>0$, there exists a constant $C=C\left(\delta,\left.\left\|\nabla_{t, \mathbf{x}} \mathbf{n}\right\|\right|_{L^{\infty}},\left\|\nabla \mathbf{n}_{t}\right\|_{L^{\infty}}\right)$ such that for any $Q \in \mathbb{M}_{0}^{3}$, it holds that

$$
\begin{aligned}
\frac{1}{\varepsilon}\left\langle\partial_{t}(\mathbf{n n}) \cdot Q, Q\right\rangle & \leq \delta\left\|\frac{1}{\varepsilon} \mathscr{H}_{\mathbf{n}}^{\varepsilon}(Q)\right\|_{L^{2}}^{2}+C_{\delta}\left(\frac{1}{\varepsilon}\left\langle\mathscr{H}_{\mathbf{n}}^{\varepsilon}(Q), Q\right\rangle+\|Q\|_{L^{2}}^{2}\right), \\
\frac{1}{\varepsilon}\left\langle Q: \partial_{t}(\mathbf{n n}), Q: \mathbf{n n}\right\rangle & \leq \delta\left\|\frac{1}{\varepsilon} \mathscr{H}_{\mathbf{n}}^{\varepsilon}(Q)\right\|_{L^{2}}^{2}+C_{\delta}\left(\frac{1}{\varepsilon}\left\langle\mathscr{H}_{\mathbf{n}}^{\varepsilon}(Q), Q\right\rangle+\|Q\|_{L^{2}}^{2}\right) .
\end{aligned}
$$

Proof. The proof of Lemma 4 can be found in [5].

Lemma 5. There holds

$$
\begin{aligned}
&\left\|Q_{R}\right\|_{H^{1}}+\left\|\left(\varepsilon \nabla^{2} Q_{R}, \varepsilon^{2} \nabla^{3} Q_{R}\right)\right\|_{L^{2}}+\left\|\left(\mathbf{v}_{R}, \varepsilon \nabla \mathbf{v}_{R}, \varepsilon^{2} \nabla^{2} \mathbf{v}_{R}\right)\right\|_{L^{2}} \leq C \mathfrak{E}^{\frac{1}{2}}, \\
&\left\|\left(\frac{1}{\varepsilon} \mathscr{H}_{\mathbf{n}}^{\varepsilon}\left(Q_{R}\right), \nabla \mathscr{H}_{\mathbf{n}}^{\varepsilon}\left(Q_{R}\right), \varepsilon \Delta \mathscr{H}_{\mathbf{n}}^{\varepsilon}\left(Q_{R}\right)\right)\right\|_{L^{2}} \leq C(\mathfrak{E}+\mathfrak{F})^{\frac{1}{2}}, \\
&\left\|\left(\varepsilon \nabla \mathscr{L}\left(Q_{R}\right), \varepsilon^{2} \Delta \mathscr{L}\left(Q_{R}\right)\right)\right\|_{L^{2}}+\left\|\left(\nabla \mathbf{v}_{R}, \varepsilon \nabla^{2} \mathbf{v}_{R}, \varepsilon^{2} \nabla^{3} \mathbf{v}_{R}\right)\right\|_{L^{2}} \leq C(\mathfrak{E}+\mathfrak{F})^{\frac{1}{2}} .
\end{aligned}
$$

Proof. The proof of Lemma 5 refers to [4,5] for details.

Corollary 1. $E \leq C \mathfrak{E}^{1 / 2}, F \leq C(\mathfrak{E}+\mathfrak{F})^{1 / 2}$.

Based on the above preliminaries, we now give the proof of Proposition 5.

Proof of Proposition 5. First, we give the following cancellation relation: for any symmetry matrix $M \in \mathbb{R}^{3 \times 3}$, there holds

$$
\begin{aligned}
- & \left\langle\chi M-Q_{0} \cdot M+M \cdot Q_{0}, \nabla \mathbf{v}_{R}\right\rangle \\
& +\left\langle\chi \mathbf{D}_{R}+\boldsymbol{\Omega}_{R} \cdot Q_{0}-Q_{0} \cdot \boldsymbol{\Omega}_{R}, M\right\rangle=0 .
\end{aligned}
$$

The proof is divided into four parts as follows:

Step 1. $L^{2}$-estimate. From the system of remainder (74)-(76) and Lemma 5, we have

$$
\begin{aligned}
& \left\langle\frac{\partial Q_{R}}{\partial t}, Q_{R}\right\rangle+\frac{1}{\mu_{1} \varepsilon}\left\langle\mathscr{H}_{\mathbf{n}}^{\varepsilon}\left(Q_{R}\right), Q_{R}\right\rangle=\left\langle\chi \mathbf{D}_{R}+\boldsymbol{\Omega}_{R} \cdot Q_{0}-Q_{0} \cdot \Omega_{R}, Q_{R}\right\rangle+\left\langle\mathfrak{R}, Q_{R}\right\rangle \\
& \quad \leq C\left\|Q_{R}\right\|_{L^{2}}\left(\left\|\nabla \mathbf{v}_{R}\right\|_{L^{2}}+\|\mathfrak{R}\|_{L^{2}}\right) \leq \delta_{0} \mathfrak{F}+C_{\delta_{0}} \mathfrak{E}+C\|\Re\|_{L^{2}}^{2} .
\end{aligned}
$$


and

$$
\begin{aligned}
\left\langle\frac{\partial \mathbf{v}_{R}}{\partial t}, \mathbf{v}_{R}\right\rangle+\left\langle\frac{\partial Q_{R}}{\partial t}, \frac{1}{\varepsilon} \mathscr{H}_{\mathbf{n}}^{\varepsilon}\left(Q_{R}\right)\right\rangle \\
=-\frac{1}{\varepsilon}\left\langle\chi \mathscr{H}_{\mathbf{n}}^{\varepsilon}\left(Q_{R}\right)-Q_{0} \cdot \mathscr{H}_{\mathbf{n}}^{\varepsilon}\left(Q_{R}\right)+\mathscr{H}_{\mathbf{n}}^{\varepsilon}\left(Q_{R}\right) \cdot Q_{0}, \nabla \mathbf{v}_{R}\right\rangle+\left\langle\nabla \cdot \mathfrak{R}+\mathfrak{R}, \mathbf{v}_{R}\right\rangle \\
\quad-\left\langle\beta_{1}^{\prime} Q_{0}\left(Q_{0}: \mathbf{D}_{R}\right)+\beta_{2}^{\prime} \mathbf{D}_{R}+\beta_{3}^{\prime}\left(Q_{0} \cdot \mathbf{D}_{R}+\mathbf{D}_{R} \cdot Q_{0}\right), \nabla \mathbf{v}_{R}\right\rangle \\
\quad-\frac{1}{\mu_{1} \varepsilon^{2}}\left\langle\mathscr{H}_{\mathbf{n}}^{\varepsilon}\left(Q_{R}\right), \mathscr{H}_{\mathbf{n}}^{\varepsilon}\left(Q_{R}\right)\right\rangle+\left\langle\chi \mathbf{D}_{R}+\mathbf{\Omega}_{R} \cdot Q_{0}-Q_{0} \cdot \Omega_{R} \frac{1}{\varepsilon} \mathscr{H}_{\mathbf{n}}^{\varepsilon}\left(Q_{R}\right)\right\rangle \\
\quad+\left\langle\Re, \frac{1}{\varepsilon} \mathscr{H}_{\mathbf{n}}^{\varepsilon}\left(Q_{R}\right)\right\rangle \\
=-s^{2} \beta_{1}^{\prime}\left\|\mathbf{n} \mathbf{n}: \mathbf{D}_{R}\right\|_{L^{2}}^{2}-\left(\beta_{2}^{\prime}-\frac{2}{3} s \beta_{3}^{\prime}\right)\left\|\mathbf{D}_{R}\right\|_{L^{2}}^{2}-2 s \beta_{3}^{\prime}\left\|\mathbf{n} \cdot \mathbf{D}_{R}\right\|_{L^{2}}^{2} \\
\quad-\frac{1}{\mu_{1} \varepsilon^{2}}\left\langle\mathscr{H}_{\mathbf{n}}^{\varepsilon}\left(Q_{R}\right), \mathscr{H}_{\mathbf{n}}^{\varepsilon}\left(Q_{R}\right)\right\rangle+\left\langle\nabla \cdot \mathfrak{R}+\mathfrak{R}, \mathbf{v}_{R}\right\rangle+\left\langle\mathfrak{R}, \frac{1}{\varepsilon} \mathscr{H}_{\mathbf{n}}^{\varepsilon}\left(Q_{R}\right)\right\rangle,
\end{aligned}
$$

where we have already used the following cancelation relation

$$
\begin{aligned}
& -\frac{1}{\varepsilon}\left\langle\chi \mathscr{H}_{\mathbf{n}}^{\varepsilon}\left(Q_{R}\right)-Q_{0} \cdot \mathscr{H}_{\mathbf{n}}^{\varepsilon}\left(Q_{R}\right)+\mathscr{H}_{\mathbf{n}}^{\varepsilon}\left(Q_{R}\right) \cdot Q_{0}, \nabla \mathbf{v}_{R}\right\rangle \\
& \quad+\left\langle\chi \mathbf{D}_{R}+\Omega_{R} \cdot Q_{0}-Q_{0} \cdot \Omega_{R}, \frac{1}{\varepsilon} \mathscr{H}_{\mathbf{n}}^{\varepsilon}\left(Q_{R}\right)\right\rangle=0 .
\end{aligned}
$$

Therefore, noting the following dissipation inequality

$$
-s^{2} \beta_{1}^{\prime}\left\|\mathbf{n n}: \mathbf{D}_{R}\right\|_{L^{2}}^{2}-\left(\frac{2}{3} \beta_{2}^{\prime}-\frac{2}{3} s \beta_{3}^{\prime}\right)\left\|\mathbf{D}_{R}\right\|_{L^{2}}^{2}-2 s \beta_{3}^{\prime}\left\|\mathbf{n} \cdot \mathbf{D}_{R}\right\|_{L^{2}}^{2} \leq 0,
$$

we can get

$$
\begin{aligned}
& \left\langle\frac{\partial \mathbf{v}_{R}}{\partial t}, \mathbf{v}_{R}\right\rangle+\left\langle\frac{\partial Q_{R}}{\partial t}, \frac{1}{\varepsilon} \mathscr{H}_{\mathbf{n}}^{\varepsilon}\left(Q_{R}\right)\right\rangle+\frac{1}{\mu_{1} \varepsilon^{2}}\left\langle\mathscr{H}_{\mathbf{n}}^{\varepsilon}\left(Q_{R}\right), \mathscr{H}_{\mathbf{n}}^{\varepsilon}\left(Q_{R}\right)\right\rangle+\frac{1}{3} \beta_{2}^{\prime}\left\|\mathbf{D}_{R}\right\|_{L^{2}}^{2} \\
& \quad \leq \delta_{0} \mathfrak{F}+C \mathfrak{E}+C\|\Re\|_{L^{2}}^{2} .
\end{aligned}
$$

Step 2. $H^{1}$-estimate. Using (74)-(76), we have

$$
\begin{aligned}
\varepsilon^{2}\langle & \left.\frac{\partial}{\partial t} \partial_{i} \mathbf{v}_{R}, \partial_{i} \mathbf{v}_{R}\right\rangle+\varepsilon\left\langle\frac{\partial}{\partial t} \partial_{i} Q_{R}, \mathscr{H}_{\mathbf{n}}^{\varepsilon}\left(\partial_{i} Q_{R}\right)\right\rangle \\
= & -\varepsilon\left\langle\partial_{i}\left[\chi \mathscr{H}_{\mathbf{n}}^{\varepsilon}\left(Q_{R}\right)-Q_{0} \cdot \mathscr{H}_{\mathbf{n}}^{\varepsilon}\left(Q_{R}\right)+\mathscr{H}_{\mathbf{n}}^{\varepsilon}\left(Q_{R}\right) \cdot Q_{0}\right], \nabla \partial_{i} \mathbf{v}_{R}\right\rangle \\
& -\varepsilon^{2}\left\langle\partial_{i}\left[\beta_{1}^{\prime} Q_{0}\left(Q_{0}: \mathbf{D}_{R}\right)+\beta_{2}^{\prime} \mathbf{D}_{R}+\beta_{3}^{\prime}\left(Q_{0} \cdot \mathbf{D}_{R}+\mathbf{D}_{R} \cdot Q_{0}\right)\right], \nabla \partial_{i} \mathbf{v}_{R}\right\rangle \\
& -\frac{1}{\mu_{1}}\left\langle\partial_{i} \mathscr{H}_{\mathbf{n}}^{\varepsilon}\left(Q_{R}\right), \mathscr{H}_{\mathbf{n}}^{\varepsilon}\left(\partial_{i} Q_{R}\right)\right\rangle+\varepsilon\left\langle\partial_{i}\left[\chi \mathbf{D}_{R}+\mathbf{\Omega}_{R} \cdot Q_{0}-Q_{0} \cdot \Omega_{R}\right], \mathscr{H}_{\mathbf{n}}^{\varepsilon}\left(\partial_{i} Q_{R}\right)\right\rangle \\
& +\varepsilon^{2}\left\langle\nabla \cdot \partial_{i} \mathfrak{R}+\partial_{i} \Re, \partial_{i} \mathbf{v}_{R}\right\rangle+\varepsilon\left\langle\partial_{i} \Re, \mathscr{H}_{\mathbf{n}}^{\varepsilon}\left(\partial_{i} Q_{R}\right)\right\rangle \\
& \stackrel{\text { def }}{=}
\end{aligned}
$$


The terms on the right-hand sides can be estimated as follows:

$$
\begin{aligned}
I \leq & -\left\langle\chi \partial_{i} \mathscr{H}_{\mathbf{n}}^{\varepsilon}\left(Q_{R}\right)-Q_{0} \cdot \partial_{i} \mathscr{H}_{\mathbf{n}}^{\varepsilon}\left(Q_{R}\right)+\partial_{i} \mathscr{H}_{\mathbf{n}}^{\varepsilon}\left(Q_{R}\right) \cdot Q_{0}, \varepsilon \nabla \partial_{i} \mathbf{v}_{R}\right\rangle \\
& +C\left\|\mathscr{H}_{\mathbf{n}}^{\varepsilon}\left(Q_{R}\right)\right\|_{L^{2}}\left\|\varepsilon \Delta \mathbf{v}_{R}\right\|_{L^{2}} \\
\leq & -\left\langle\chi \mathscr{H}_{\mathbf{n}}^{\varepsilon}\left(\partial_{i} Q_{R}\right)-Q_{0} \cdot \mathscr{H}_{\mathbf{n}}^{\varepsilon}\left(\partial_{i} Q_{R}\right)+\mathscr{H}_{\mathbf{n}}^{\varepsilon}\left(\partial_{i} Q_{R}\right) \cdot Q_{0}, \varepsilon \nabla \partial_{i} \mathbf{v}_{R}\right\rangle \\
& +\delta_{0} \mathfrak{F}+C \mathfrak{E}, \\
I I \leq & -\varepsilon^{2}\left\langle\beta_{1}^{\prime} Q_{0}\left(Q_{0}: \partial_{i} \mathbf{D}_{R}\right)+\beta_{2}^{\prime} \partial_{i} \mathbf{D}_{R}+\beta_{3}^{\prime}\left(Q_{0} \cdot \partial_{i} \mathbf{D}_{R}+\partial_{i} \mathbf{D}_{R} \cdot Q_{0}\right), \nabla \partial_{i} \mathbf{v}_{R}\right\rangle \\
& +C\left\|\varepsilon \nabla \mathbf{v}_{R}\right\|_{L^{2}}\left\|\varepsilon \nabla \partial_{i} \mathbf{v}_{R}\right\|_{L^{2}} \\
\leq & -\varepsilon^{2} s^{2} \beta_{1}^{\prime}\left\|\mathbf{n n}: \partial_{i} \mathbf{D}_{R}\right\|_{L^{2}}^{2}-\varepsilon^{2}\left(\beta_{2}^{\prime}-\frac{2}{3} s \beta_{3}^{\prime}\right)\left\|\partial_{i} \mathbf{D}_{R}\right\|_{L^{2}}^{2}-2 \varepsilon^{2} s \beta_{3}^{\prime}\left\|\mathbf{n} \cdot \partial_{i} \mathbf{D}_{R}\right\|_{L^{2}}^{2} \\
& +\delta_{0} \mathfrak{F}+C \mathfrak{E}, \\
I I I \leq & -\frac{1}{\mu_{1}}\left\|\mathscr{H}_{\mathbf{n}}^{\varepsilon}\left(\partial_{i} Q_{R}\right)\right\|_{L^{2}}^{2}+C\left\|Q_{R}\right\|_{L^{2}}\left\|\mathscr{H}_{\mathbf{n}}^{\varepsilon}\left(Q_{R}\right)\right\|_{L^{2}} \\
\leq & -\frac{1}{\mu_{1}}\left\|\mathscr{H}_{\mathbf{n}}^{\varepsilon}\left(\partial_{i} Q_{R}\right)\right\|_{L^{2}}^{2}+\delta_{0} \mathfrak{F}+C \mathfrak{E}, \\
I V \leq & \varepsilon\left\langle\chi \partial_{i} \mathbf{D}_{R}+\partial_{i} \Omega_{R} \cdot Q_{0}-Q_{0} \cdot \partial_{i} \Omega_{R}, \mathscr{H}_{\mathbf{n}}^{\varepsilon}\left(\partial_{i} Q_{R}\right)\right\rangle+\delta_{0} \mathfrak{F}+C \mathfrak{E}, \\
V \leq & \left\|\varepsilon \partial_{i} \mathfrak{R}\right\|_{L^{2}}\left\|\varepsilon \nabla \partial_{i} \mathbf{v}_{R}\right\|_{L^{2}}+\left\|\varepsilon \partial_{i} \mathfrak{R}\right\|_{L^{2}}\left\|\varepsilon \partial_{i} \mathbf{v}_{R}\right\|_{L^{2}} \\
\leq & \delta_{0} \mathfrak{F}+C \mathfrak{E}+C \varepsilon_{\varepsilon} \partial_{i} \|_{L^{2}}^{2} \\
V I \leq & \left\|\varepsilon \partial_{i} \mathfrak{R}\right\|_{L^{2}}\left\|\mathscr{H}_{\mathbf{n}}^{\varepsilon}\left(\partial_{i} Q_{R}\right)\right\|_{L^{2}} \leq \delta_{0} \mathfrak{F}+C\left\|\varepsilon \partial_{i} \mathfrak{R}\right\|_{L^{2}}^{2} .
\end{aligned}
$$

Therefore, by the cancelation relation (80) and the dissipation inequality similar to that in Step 2, we have

$$
\begin{aligned}
& \varepsilon^{2}\left\langle\frac{\partial}{\partial t} \partial_{i} \mathbf{v}_{R}, \partial_{i} \mathbf{v}_{R}\right\rangle+\varepsilon\left\langle\frac{\partial}{\partial t} \partial_{i} Q_{R}, \mathscr{H}_{\mathbf{n}}^{\varepsilon}\left(\partial_{i} Q_{R}\right)\right\rangle \\
& \quad \leq-\varepsilon^{2} \frac{1}{3} \beta_{2}^{\prime}\left\|\partial_{i} \mathbf{D}_{R}\right\|_{L^{2}}^{2}-\frac{1}{\mu_{1}}\left\|\mathscr{H}_{\mathbf{n}}^{\varepsilon}\left(\partial_{i} Q_{R}\right)\right\|_{L^{2}}^{2}+\delta_{0} \mathfrak{F}+C \mathfrak{E}+C\left\|\varepsilon \partial_{i} \Re\right\|_{L^{2}}^{2} .
\end{aligned}
$$

Step 3. $H^{2}$-estimate. Using (74)-(76) and emulating the proof of Step 2, it is easy to obtain the corresponding $H^{2}$-estimate:

$$
\begin{aligned}
& \varepsilon^{4}\left\langle\frac{\partial}{\partial t} \Delta \mathbf{v}_{R}, \Delta \mathbf{v}_{R}\right\rangle+\varepsilon^{2}\left\langle\frac{\partial}{\partial t} \Delta Q_{R}, \mathscr{H}_{\mathbf{n}}^{\varepsilon}\left(\Delta Q_{R}\right)\right\rangle \\
& \quad \leq-\varepsilon^{4} \frac{1}{3} \beta_{2}^{\prime}\left\|\Delta \mathbf{D}_{R}\right\|_{L^{2}}^{2}-\frac{\varepsilon^{2}}{\mu_{1}}\left\|\mathscr{H}_{\mathbf{n}}^{\varepsilon}\left(\Delta Q_{R}\right)\right\|_{L^{2}}^{2}+\delta_{0} \mathfrak{F}+C \mathfrak{E}+C\|\varepsilon \Delta \Re\|_{L^{2}}^{2} .
\end{aligned}
$$

Step 4. Closure of error estimates. Noting the definition of $\mathscr{H}_{\mathbf{n}}^{\varepsilon}\left(Q_{R}\right)$, from Lemma 4, it follows that

$$
\begin{aligned}
\frac{1}{\varepsilon} \frac{d}{d t} & \left\langle Q_{R}, \mathscr{H}_{\mathbf{n}}^{\varepsilon}\left(Q_{R}\right)\right\rangle \\
= & \frac{2}{\varepsilon}\left\langle\frac{\partial}{\partial t} Q_{R}, \mathscr{H}_{\mathbf{n}}^{\varepsilon}\left(Q_{R}\right)\right\rangle+\frac{1}{\varepsilon}\left\langle Q_{R},-2 c s^{2}\left(\mathbf{n n}: Q_{R}\right) \partial_{t}(\mathbf{n n})-2 c s^{2}\left(\partial_{t}(\mathbf{n n}): Q_{R}\right) \mathbf{n n}\right. \\
& \left.+b s\left(\partial_{t}(\mathbf{n n}) \cdot Q_{R}+Q_{R} \cdot \partial_{t}(\mathbf{n n})\right)\right\rangle \\
= & \frac{2}{\varepsilon}\left\langle\frac{\partial}{\partial t} Q_{R}, \mathscr{H}_{\mathbf{n}}^{\varepsilon}\left(Q_{R}\right)\right\rangle+\frac{2}{\varepsilon}\left\langle Q_{R},-2 c s^{2}\left(\partial_{t}(\mathbf{n n}): Q_{R}\right) \mathbf{n n}+b s \partial_{t}(\mathbf{n n}) \cdot Q_{R}\right\rangle \\
\leq & \frac{2}{\varepsilon}\left\langle\frac{\partial}{\partial t} Q_{R}, \mathscr{H}_{\mathbf{n}}^{\varepsilon}\left(Q_{R}\right)\right\rangle+\delta\left\|\frac{1}{\varepsilon} \mathscr{H}_{\mathbf{n}}^{\varepsilon}\left(Q_{R}\right)\right\|_{L^{2}}^{2}+C_{\delta}\left(\frac{1}{\varepsilon}\left\langle\mathscr{H}_{\mathbf{n}}^{\varepsilon}\left(Q_{R}\right), Q_{R}\right\rangle+\left\|Q_{R}\right\|_{L^{2}}^{2}\right) .
\end{aligned}
$$


Then we obtain

$$
\frac{1}{2 \varepsilon} \frac{d}{d t}\left\langle Q_{R}, \mathscr{H}_{\mathbf{n}}^{\mathcal{\varepsilon}}\left(Q_{R}\right)\right\rangle \leq\left\langle\frac{\partial}{\partial t} Q_{R}, \mathscr{H}_{\mathbf{n}}^{\varepsilon}\left(Q_{R}\right)\right\rangle+\delta \mathfrak{F}+C \mathfrak{E} .
$$

A similar method leads to the following inequalities:

$$
\begin{aligned}
& \frac{\varepsilon}{2} \frac{d}{d t}\left\langle\partial_{i} Q_{R}, \mathscr{H}_{\mathbf{n}}^{\varepsilon}\left(\partial_{i} Q_{R}\right)\right\rangle \leq \varepsilon\left\langle\frac{\partial}{\partial t} \partial_{i} Q_{R}, \mathscr{H}_{\mathbf{n}}^{\varepsilon}\left(\partial_{i} Q_{R}\right)\right\rangle+\delta \mathfrak{F}+C \mathfrak{E}, \\
& \frac{\varepsilon^{3}}{2} \frac{d}{d t}\left\langle\Delta Q_{R}, \mathscr{H}_{\mathbf{n}}^{\varepsilon}\left(\Delta Q_{R}\right)\right\rangle \leq \varepsilon^{3}\left\langle\frac{\partial}{\partial t} \Delta Q_{R}, \mathscr{H}_{\mathbf{n}}^{\varepsilon}\left(\Delta Q_{R}\right)\right\rangle+\delta \mathfrak{F}+C \mathfrak{E} .
\end{aligned}
$$

Therefore, together with (81)-(84), by using the property of good terms $\mathfrak{R}$ and Corollary 1 , we arrive at

$$
\begin{aligned}
\frac{1}{2} \frac{d}{d t} \mathfrak{E}(t)+\mathfrak{F}(t) & \leq \delta \mathfrak{F}+C_{\delta} \mathfrak{E}+\|\mathfrak{R}\|_{L^{2}}^{2}+\|\varepsilon \nabla \mathfrak{R}\|_{L^{2}}^{2}+\left\|\varepsilon^{2} \Delta \mathfrak{R}\right\|_{L^{2}}^{2} \\
& \leq \delta \mathfrak{F}+C_{\delta} \mathfrak{E}+C(\varepsilon E)(1+E+\varepsilon F)+\varepsilon f(E) \\
& \leq \delta \mathfrak{F}+C_{\delta} \mathfrak{E}+C\left(\varepsilon^{2} \mathfrak{E}\right)\left(1+\mathfrak{E}+\varepsilon^{2} \mathfrak{F}\right)+\varepsilon^{2} f(\mathfrak{E}),
\end{aligned}
$$

which concludes the proof of Proposition 5 by taking small enough $\delta$.

\section{Conclusions}

This article is mainly concerned with the non-inertial Qian-Sheng model describing nematic liquid crystal flows. In the framework of smooth solutions, by taking the elastic constants tend to zero and using the Hilbert expansion, we rigorously prove that the solution to the non-inertial Qian-Sheng model converges to the solution to the EricksenLeslie model. The original Qian-Sheng model, in which the inertial term is responsible for the hyperbolic feature of the evolution equation of $Q$-tensor, involves two small parameters: the inertial coefficient and the elastic coefficient. In recent work [7], the inertial coefficient is fixed and the singular limit (also called the unixial limit) of the original model is rigorously investigated. A natural problem is how to understand the singular limit of the original model when the two small parameters go to zero at the same time. It is natural to conjecture that by taking the two parameters tend to zero, the smooth solution to the original model will converge to the solution to the non-inertial Ericksen-Leslie model. This is an interesting problem which will be discussed, while this article paves the way for our future work.

Author Contributions: All authors contributed to the draft of the manuscript. All authors have read and agreed to the published version of the manuscript.

Funding: Sirui Li is supported by the NSF of China under Grant No. 12061019, and by the Growth Foundation for Youth Science and Technology Talent of Educational Commission of Guizhou Province of China under Grant No. [2021]087.

Institutional Review Board Statement: Not applicable.

Informed Consent Statement: Not applicable.

Data Availability Statement: Not applicable.

Acknowledgments: The authors thank the anonymous referees and editor for their valuable suggestion and comments to improve this work.

Conflicts of Interest: The authors declare no conflict of interest to the work.

\section{References}

1. Kuzuu, N.; Doi, M. Constitutive equation for nematic liquid crystals under weak velocity gradient derived from a molecular kinetic equation. J. Phys. Soc. Jpn. 1983, 52, 3486-3494. [CrossRef]

2. Zhang, W.E.P. A molecular kinetic theory of inhomogeneous liquid crystal flow and the small Deborah number limit. Methods Appl. Anal. 2006, 13, 181-198. 
3. Wang, W.; Zhang, P.; Zhang, Z. The small Deborah number limit of the Doi-Onsager equation to the Ericksen-Leslie equation. Commun. Pure Appl. Math. 2015, 68, 1326-1398. [CrossRef]

4. Li, S.-R.; Wang, W.; Zhang, P. Local well-posedness and small Deborah limit of a molecule-based Q-tensor system. Discret. Contin. Dyn. Syst. Ser. B 2015, 20, 2611-2655. [CrossRef]

5. Wang, W.; Zhang, P.; Zhang, Z. Rigorous derivation from Landau-de Gennes theory to Ericksen-Leslie theory. SIAM J. Math. Anal. 2015, 47, 127-158. [CrossRef]

6. Han, J.; Luo, Y.; Wang, W.; Zhang, P.; Zhang, Z. From microscopic theory to macroscopic theory: Systematic study on modeling for liquid crystals. Arch. Ration. Mech. Anal. 2015, 215, 741-809. [CrossRef]

7. Li, S.-R.; Wang, W. Rigorous justification of the uniaxial limit from the Qian-Sheng inertial Q-tensor theory to the Ericksen-Lesile theory. SIAM J. Math. Anal. 2020, 52, 4421-4468. [CrossRef]

8. Popa-Nita, V.; Sluckin, T.J.; Kralj, S. Waves at the nematic-isotropic interface: Thermotropic nematogen-nonnematogen mixtures. Phys. Rev. E 2005, 71, 061706. [CrossRef]

9. Svensek, D.; Zummer, S. Hydrodynamics of pair-annihilating disclination lines in nematic liquid crystals. Phys. Rev. E 2002, 66, 021712-021716. [CrossRef]

10. Ericksen, J. Conservation laws for liquid crystals. Trans. Soc. Rheol. 1961, 5, 22-34. [CrossRef]

11. Leslie, F.M. Some constitutive equations for liquid crystals. Arch. Ration. Mech. Anal. 1968, 28, 265-283. [CrossRef]

12. Lin, F.-H.; Liu, C. Existence of solutions for the Ericksen-Leslie system. Arch. Ration. Mech. Anal. 2000, 154, 135-156. [CrossRef]

13. Lin, F.-H.; Liu, C. Static and dynamical theories of liquid crystals. J. Partial Differ. Equ. 2001, 14, $289-330$.

14. Lin, F.-H.; Wang, C. Recent developments of analysis for hydrodynamic flow of nematic liquid crystals. Philos. Trans. R. Soc. A 2014, 372, 20130361. [CrossRef]

15. Wang, W.; Zhang, P.; Zhang, Z. Well-posedness of the Ericksen-Leslie system. Arch. Ration. Mech. Anal. 2012, 206, 953-995. [CrossRef]

16. Jiang, N.; Luo, Y. On well-posedness of Ericksen-Leslie's hyperbolic incompressible liquid crystal model. SIAM J. Math. Anal. 2019, 51, 403-434. [CrossRef]

17. Cai, Y.; Wang, W. Global well-posedness for the three dimensional simplified inertial Ericksen-Leslie systems near equilibrium. J. Funct. Anal. 2020, 279, 108521. [CrossRef]

18. Huang, J.; Jiang, N.; Luo, Y.-L.; Zhao, L. Small data global regularity for the 3-D Ericksen-Leslie hyperbolic liquid crystal model without kinematic transport. SIAM J. Math. Anal. 2021, 53, 530-573. [CrossRef]

19. De Gennes, P.G. The Physics of Liquid Crystals; Clarendon Press: Oxford, UK, 1974.

20. Mottram, N.J.; Newton, C. Introduction to Q-tensor theory. arXiv 2014, arXiv:1409.3542.

21. Beris, A.N.; Edwards, B.J. Thermodynamics of Flowing Systems with Internal Microstructure; Oxford Engrg. Sci. Ser. 36; Oxford University Press: Oxford, UK; New York, NY, USA, 1994.

22. Qian, T.; Sheng, P. Generalized hydrodynamic equations for nematic liquid crystals. Phys. Rev. E 1998, 58, 7475-7485. [CrossRef]

23. Paicu, M.; Zarnescu, A. Energy dissipation and regularity for a coupled Navier-Stokes and Q-tensor system. Arch. Ration. Mech. Anal. 2012, 203, 45-67. [CrossRef]

24. Paicu, M.; Zarnescu, A. Global existence and regularity for the full coupled Navier-Stokes and Q-tensor system. SIAM J. Math. Anal. 2011,43, 2009-2049. [CrossRef]

25. Huang, J.; Ding, S. Global well-posedness for the dynamical Q-tensor model of liquid crystals. Sci. China Math. 2015, 58, 1349-1366. [CrossRef]

26. Abels, H.; Dolzmann, G.; Liu, Y. Well-posedness of a fully-coupled Navier-Stokes/Q-tensor system with inhomogeneous boundary data. SIAM J. Math. Anal. 2014, 46, 3050-3077. [CrossRef]

27. Abels, H.; Dolzmann, G.; Liu, Y. Strong solutions for the Beris-Edwards model for nematic liquid crystals with homogeneous Dirichilet boundary conditions. Adv. Differ. Equ. 2016, 21, 109-152.

28. Liu, Y.; Wang, W. On the initial boundary value problem of a Navier-Stokes/Q-tensor model for liquid crystals. Discret. Contin. Dyn. Syst. Ser. B 2018, 23, 3879-3899. [CrossRef]

29. De Anna, F.; Zarnescu, A. Global well-posedness and twist-wave solutions for the inertial Qian-Sheng model of liquid crystals. J. Differ. Equ. 2018, 264, 1080-1118. [CrossRef]

30. Feireisl, E.; Rocca, E.; Schimperna, G.; Zarnescu, A. On a hyperbolic system arising in liquid crystals modeling. J. Hyperbolic Differ. Equ. 2018, 15, 15-35. [CrossRef]

31. Ma, Y. Global well-posedness to incompressible non-inertial Qian-Sheng model. Discret. Contin. Dyn. Syst. 2020, 40, 4479-4496. [CrossRef]

32. Parodi, O. Stress tensor for a nematic liquid crystal. J. Phys. 1970, 31, 581-584. [CrossRef]

33. Majumdar, A. Equilibrium order parameters of nematic liquid crystals in the Landau-de Gennes theory. Eur. J. Appl. Math. 2010, 21, 181-203. [CrossRef] 